\title{
Shear effects on crystal nucleation in colloidal suspensions
}

\author{
Juan J. Cerdà, ${ }^{1}$ Tomás Sintes, ${ }^{2}$ C. Holm, ${ }^{1,3}$ C. M. Sorensen, ${ }^{4}$ and A. Chakrabarti ${ }^{4}$ \\ ${ }^{1}$ Frankfurt Institute for Advanced Studies, J. W. Goethe-Universität, Ruth-Moufang-Strasse 1, D-60438, Frankfurt am Main, Germany \\ ${ }^{2}$ Instituto de Física Interdisciplinar y Sistemas Complejos IFISC (CSIC-UIB), Universitat de les Illes Balears, \\ 07122 Palma de Mallorca, Spain \\ ${ }^{3}$ Max-Planck-Institut für Polymerforschung, Ackermannweg 10, D-55128, Mainz, Germany \\ ${ }^{4}$ Department of Physics, Kansas State University, Cardwell Hall, Manhattan, Kansas 66506-2601, USA \\ (Received 5 April 2008; published 8 September 2008)
}

\begin{abstract}
Extensive two-dimensional Langevin dynamics simulations are used to determine the effect of steady shear flows on the crystal nucleation kinetics of charge stabilized colloids and colloids whose pair potential possess an attractive shallow well of a few $k_{B} T$ 's (attractive colloids). Results show that in both types of systems small amounts of shear speeds up the crystallization process and enhances the quality of the growing crystal significantly. Moderate shear rates, on the other hand, destroy the ordering in the system. The very high shear rate regime where a reentering transition to the ordered state could exist is not considered in this work. In addition to the crystal nucleation phenomena, the analysis of the transport properties and the characterization of the steady state regime under shear are performed.
\end{abstract}

DOI: 10.1103/PhysRevE.78.031403 PACS number(s): 82.70.-y, 64.60.qe, 64.60.Cn, 64.70.pv

\section{INTRODUCTION}

The kinetics of crystallization in colloidal systems is relevant to a large number of technological and medical applications in addition to their importance as a fundamental problem in nonequilibrium statistical mechanics. The ability to control the rate of crystallization and the final morphology of crystals formed is crucial in many technological applications. A better control could lead, for instance, to more efficient nanostructured photonic materials [1-3] and improved pharmaceutical products [4]. In addition, a better determination of the crystal structure of proteins [5], or a better control of protein aggregation would be important in the treatment of diseases such as sickle cell anaemia or cataract [6-8] among others.

The active control of crystallization of colloids using external fields has been the subject of a large number of studies: Acoustic [9], hydrodynamic [10], gravitational [11]; electric and magnetic fields, optical tweezers, as well as shear (for a review see Refs. [12-16]). In particular, studies of shear flows imposed on systems that exhibit orderdisorder transitions have led to the discovery of several shear-induced phase transitions: Shear-induced melting $[17,18]$, phase segregation, liquid-crystal transitions, and shear-induced ordering $[19,20]$. These nonequilibrium transitions are of fundamental interest and essential to understand phenomena such as wear and lubrication [21]. The effect of shear in systems confined between parallel plates, and the relaxation back to equilibrium from a sheared steady state has been addressed recently [22-25].

The effect of shear flow on the ordering of colloidal systems has recently been reviewed by Vermant et al. [26]. It turns out that while the effects of high shear rates on crystallization are quite well understood, a thorough understanding of the role that moderate and low shear rates play on order-disorder transitions is still lacking. For example, the off-critical part of the gas-liquid spinodal of colloids has been predicted to displace linearly with the shear rate [27], but the question of whether shear increases or decreases the spinodal temperature remains open and seems to depend on the nature of each specific system. Several experiments [28-33] have suggested that shearing can promote or speed up the crystallization process in colloids, while other experiments report shear to hamper $[34,35]$, or do not make a significant influence on crystallization [36]. In numerical simulations where charge-stabilized colloidal systems (interacting via a repulsive Yukawa pair potential) have been used $[37,38]$, the main conclusions are that shear suppresses crystal nucleation for small and moderate shear rates. Very recently, Mokshin-Barrat [39] have reported moderate amounts of shear to enhance the degree of order in their molecular dynamics simulations of glassy systems where particles interact via a repulsive potential (shifted Lennard-Jones potential). On the other hand, a recent theoretical study has predicted that in typical crystallization systems, the enhancement of transport to the emerging nucleus due to shear flow is negligible when compared to pure diffusion [40].

In order to shed light on these seemingly contradictory results, an exhaustive numerical study of the nucleation processes that take place at low to moderate shear rates in colloidal systems is performed in this paper. The study covers two major categories of colloids in terms of the overall effect of the effective pair potentials: Repulsive colloids and attractive colloids. In this paper charged-stabilized colloids interacting via a Yukawa pair potential $[13,17,37,38]$ have been selected as a model for repulsive colloids in which crystallization occurs at high densities, while the prototype of a short-range attractive model considered here is the AsakuraOosawa-Vrij potential [41] in depletion-driven colloids.

The effect of shear on attractive colloidal systems has received considerable recent attention. Several theoretical models devoted to the behavior of aggregates under shear have been developed [42-45]. The rheology and properties of clusters in the steady state have been addressed using sticky particle simulations [46], pair-drag simulations $[47,48]$, and experiments [49]. Further studies include both 
anisotropic (see Ref. [50] for a review) and isotropic interactions [42-44,47-49,51,52] and simulations in the presence of explicit solvent molecules [53]. Transformations induced by shear on gel-like aggregates of attractive colloids have also been studied using Brownian dynamics [54] and Stokesian simulations [55]. Despite previous studies though, the kinetics of the crystallization process in attractive colloids is still not well understood. In this paper, polymer-driven depletion colloids has been selected as a model to study the kinetics of crystallization under shear.

Our main results are the following. At low shear rates, shear flow is observed to enhance and speed up the process of crystal nucleation in both repulsive and attractive colloidal systems, while for moderately large shear rates crystal nucleation is suppressed. For repulsive colloids, this latter result is in agreement with previous numerical studies [17,37,39].

The rest of the paper is organized as follows. In Sec. II Langevin dynamics algorithm used to study both attractive and repulsive colloidal models is discussed. In Sec. III model and results for repulsive colloids are presented, while in Sec. IV attractive colloids are studied. Finally, Sec. V concludes with a summary of our work.

\section{NUMERICAL MODEL}

In our two-dimensional (2D) model a shear flow is introduced along the $x$ direction while the $y$ axis corresponds to the gradient direction. The solvent is implicitly represented by a local friction coefficient and random force acting on the particles. The equation of motion for the colloidal particles under shear is approached by the following Langevin equation [56]:

$$
m \ddot{\mathbf{r}}_{i}=-\nabla U_{i}-\Gamma \dot{\mathbf{r}}_{i}+\mathbf{W}_{i}(t)+\beta\left(y_{i}-\frac{L}{2}\right) \hat{e}_{x},
$$

where $U_{i}$ is the pair-interaction potential, $\Gamma$ is the friction coefficient acting on the colloidal particles, and $\hat{e}_{x}$ is the unit vector along the $x$ axis. $\mathbf{W}_{i}(t)$ describes the random force acting on each colloidal particle, and is set to be a Gaussian white noise with zero mean that satisfies the 2D fluctuationdissipation relation: $\left\langle\mathbf{W}_{i}(t) \cdot \mathbf{W}_{j}\left(t^{\prime}\right)\right\rangle_{\mathrm{eq}}=4 k_{B} T_{\mathrm{ref}} \Gamma \delta_{i j} \delta\left(t-t^{\prime}\right)$, where $\langle\cdots\rangle_{\text {eq }}$ denotes averaging over an equilibrium ensemble, $k_{B}$ is the Boltzmann constant, and $T_{\text {ref }}$ is the reference temperature. In order to model shear flow, an external force along the flow direction $\beta\left(y_{i}-\frac{L}{2}\right)$ has been imposed, being $L$ the system size, and $\beta \equiv \Gamma \dot{\gamma}$, where $\dot{\gamma}$ is the shear flow rate that we aim to obtain. Our tests show that the addition of this force produces a steady shear flow with rate $\dot{\gamma}$, and a mean velocity given by

$$
\widetilde{v}_{x}(y) \equiv \lim _{\Delta y \rightarrow 0}\left\langle v_{x}(i)\right\rangle_{[y, y+\Delta y)}=\dot{\gamma}\left(y-\frac{L}{2}\right),
$$

where $\left\langle v_{x}(i)\right\rangle_{[y, y+\Delta y)}$ means that for a given value of the $y$ coordinate, all the particles in the system with their $y$ coordinate lying in the interval $[y, y+\Delta y)$ have been considered to perform the average. All distances, masses, and times are expressed in reduced units $L / \sigma, M / m, t / \tau$, where $\sigma$ and $m$ are the particle diameter and mass, respectively (for conve- nience we set $\sigma=1$ and $m=1$ ), and $\tau=\left(\sigma m / k_{B} T_{\text {ref }}\right)^{1 / 2}$ (again for convenience we take $k_{B} T_{\text {ref }}=1$ ).

Langevin equations (1) have been integrated using the third-order algorithm of van Gunsteren-Berendsen (BDGB) [57], which allows us to use larger time steps than other usual algorithms (see discussion in [57]). Hydrodynamic interactions resulting from the solvent motion are ignored (free draining limit). Despite the fact that a complete understanding of sheared suspensions requires a full treatment of the many-body hydrodynamic interactions, we argue that at least a good qualitative insight into the effects of shear in the crystallization process is still possible using the free draining limit $[19,38]$. Nonetheless, hydrodynamic interactions are expected to increase in importance when shear rates are high, and conclusions derived from free draining simulations in the high shear rates regime must be considered with care. Furthermore, at high shear rates, the introduction of a linear force $\beta\left(y_{i}-\frac{L}{2}\right)$ in the Langevin equations 1 to mimic shear effects on colloidal particles could not represent the correct physics. Although discrete particle simulations with explicit solvent would be ideal to solve those problems, they are extremely demanding computationally. Mesoscale hydrodynamics simulations [58,59] (lattice Boltzmann, dissipative particle dynamics, multiparticle collision dynamics) although reduce considerably the computer effort with respect to explicit solvent simulations, unfortunately still are out of our computational capabilities for the system sizes we aim to study.

Periodic boundary conditions are imposed along the flow direction $x$, where Lees-Edwards boundary conditions (LEbc) [60] are used along the gradient direction $y$. The BDGB algorithm used in this work does not require the calculation of velocities, which allows us to save computer time, nonetheless this implies that LEbc must be adapted in our simulations (see the Appendix). Special care has been taken to ensure that the implementation of the algorithm, as well as the choice of the time step $d t$ leads to fluctuating part of the velocity distributions that do not depend on the distance of the particles to the boundaries, i.e., the velocity distribution of particles located between $y$ and $y+\Delta y$ does not depend on the value of $y$ once $\widetilde{v}_{x}(y)$ has been subtracted. Further details of the simulations are given in Secs. III and IV.

Both repulsive and attractive colloidal Langevin dynamics simulations are performed using a friction coefficient $\Gamma$ $=0.5$ which leads to a free diffusion coefficient $D_{o} \approx 2.35$. The Deborah number is defined as De $\equiv \dot{\gamma} \tau_{o}$, where $\tau_{o}$ $\equiv \sigma^{2} /\left(4 D_{o}\right) \approx 0.106$ is the time required for a particle to diffuse a distance $\sigma$ in absence of shear and any other particles.

\section{CHARGE STABILIZED COLLOIDS}

\section{A. System description}

Although our study is confined to two dimensions, we often compare our results with the previous work by ButlerHarrowell (BH) [17,38] carried out in three dimensions (3D). We consider a system of linear size $L=64$ containing $N_{p}$ $=4096$ colloidal particles corresponding to a number density of particles equal to unity (in analogy to $\mathrm{BH}$ simulations). 
Particles interact via screened Coulomb or Yukawa pair potential

$$
U(\mathbf{r})=\frac{V_{O}}{|\mathbf{r}|} e^{-\kappa|\mathbf{r}|},
$$

where the inverse Debye length is set to $\kappa=3.1$, and the cutoff radius is $r_{\text {cut }}=8 / \kappa$ as in the $\mathrm{BH}$ study. $V_{O}$ is varied in order to tune the degree of repulsion of the Yukawa interaction. When $V_{O}$ increases the effective particle size increases and therefore the effective volume fraction also increases. Yukawa repulsive colloids are know to show a order-disorder transition when the reduced temperature $T=V_{O} /\left(k_{B} T_{\text {ref }}\right)$ is increased [38]. In our simulations we maintain $k_{B} T_{\text {ref }}=1$, and to study the transition we increase the value of the potential parameter $V_{0}$ [Eq. (3)]. The equivalences with the reduced temperature defined in the Butler-Harrowell study, $T_{\mathrm{BH}}$ $\equiv \frac{6 k_{B} T}{V_{O}}$ are the following: Initial system equilibration in $\mathrm{BH}$ is performed at $T_{\mathrm{BH}}=0.04$, equivalent to $V_{O}=150 k_{B} T$ in our model; the melting temperature found by $\mathrm{BH}$ in their $3 \mathrm{D}$ systems is $T_{\mathrm{BH}}=0.00256$, equivalent to setting $V_{O}=250 k_{B} T$ in our model.

It should be mentioned that our aim is to study how shear affects the transition. Although by changing the number density of particles (within the crystallization regime) the transition will occur at different reduced temperatures and the time of the kinetics will be different, we do not expect our conclusions to depend on the exact chosen value of the particle density. For this reason, all our simulations are performed at the same particle number density as in the study of $\mathrm{BH}$.

The time step needed to ensure the fluctuating part of the velocity profile to be independent of the position of the particle along the $y$ direction decreases with increasing either $V_{0}$ or the shear rate $\dot{\gamma}$. For $\dot{\gamma}=0$ typical values used in simulations are in the range $d t \in\left(5 \times 10^{-3}, 5 \times 10^{-4}\right)$ for $V_{O}$ $\in(10,2000)$. For $V_{O}=2000$, the range of time steps used is $d t \in\left(5 \times 10^{-4}, 5 \times 10^{-6}\right)$ for $\dot{\gamma} \in(0,5)$.

In order to study how shear affects the kinetics of the transition, it is convenient that the initial conformation at $t$ $=0$ corresponds to a disordered state. The initial conformations are obtained using a standard Monte Carlo program as follows: Particles are distributed randomly inside the system, and random velocities following Maxwell distribution are assigned to the particles. The system is subsequently thermalized during 1000 reduced time units at zero shear rate $\dot{\gamma}=0$, and $V_{O}=10$ (BH used $V_{0}=100$ in their thermalization protocol). As we will see later, $V_{O}=10$ leads to very disordered systems, and constitutes an adequate starting point to study the transition. It should be remarked that the thermalization must be performed at $V_{O} \neq 0$ using a Monte Carlo program to prevent the problem that if two particles are very close in a random conformation, the time step needed to integrate correctly the Langevin equations is almost zero. Several tests performed using different values of $V_{O}$ during the thermalization showed that almost no differences exist in the temporal evolution of the observables when $V_{O}<100$. Once thermalization has ended, we set $t=0$ and the Langevin dynamics starts using the desired $V_{0}$ and shear rate $\dot{\gamma}$ values.
The steady mean velocity profile [Eq. (2)] is reached in a short period of time compared to the times relevant for the formation of a crystal nucleus. No differences were observed on the relevant scales of the temporal evolution of the observables when at $t=0$ an initial velocity profile similar to Eq. (2) was imposed to the particles.

In order to determine the degree of order exhibited in this Yukawa repulsive colloidal model, the global orientation order parameters $\Psi_{n}(t)$ are used. These parameters are defined as

$$
\Psi_{n}(t) \equiv\left\langle\frac{1}{N_{p}}\left|\sum_{j=1}^{N_{p}} \phi_{n, j}(t)\right|\right\rangle,
$$

where $\phi_{n, j}$ is the $n$-fold bond orientation order parameter given by

$$
\phi_{n, j}(t) \equiv \frac{1}{n_{j}(t)} \sum_{k=1}^{n_{j}} \exp \left[\operatorname{in} \theta_{j k}(t)\right],
$$

where $i \equiv \sqrt{-1}, \theta_{j k}(t)$ is the angle at time $t$ that the relative position vector of particles $j, k\left(\mathbf{r}_{j k}=\mathbf{r}_{j}-\mathbf{r}_{k}\right)$ forms with the $x$ axis, and $n_{j}(t)$ is the number of nearest neighbors of particle $j$ at time $t$. A particle $i$ is considered to be a nearest neighbor of particle $j$ if $\left|\mathbf{r}_{i j}\right|<1.4 \sigma$ which ensures that only the first shell of surrounding neighbors of a given particle will be taken into account provided that in the system under study the mean distance between neighboring particles is roughly $\sigma$. The brackets $\langle f(t)\rangle$ imply that the function $f(t)$ has been averaged over the conformations at time $t$ obtained from 20 to 50 runs with different initial conformations. Orientation order parameters for $n \in(1,8)$ have been computed, although a posteriori it has been observed that due to the relative distribution of particles in the ordered phase, $\Psi_{6}(t)$ is enough to characterize the behavior of the systems under study.

A second measure of order used in our study (closely following $\mathrm{BH}$ ) is the time evolution of the maximum of the $q_{y}$-averaged structure factor $I(t)$ defined as

$$
\begin{gathered}
I(t) \equiv\left\langle I_{\max }(t)\right\rangle, \\
I_{\max }(t) \equiv \max \left[\left\langle S\left(q_{x}, q_{y}, t\right)\right\rangle_{q_{y}}\right],
\end{gathered}
$$

where $\langle\cdots\rangle_{q_{y}}$ implies that the structure factor $S\left(q_{x}, q_{y}, t\right)$ has been averaged along the reciprocal gradient direction $q_{y}$ leading to a function $S\left(q_{x}, t\right)$ from which the maximum at each instant is determined by averaging over the conformations at time $t$ obtained from 20 to 50 runs with different initial conditions.

It should be remarked that the initial conformations obtained at $V_{O}=10$, clearly correspond to what we call a disordered state because the structure factors of the samples do not exhibit pronounced Bragg peaks. Thus $I(t)$ is very small, and the orientational order parameter $\Psi_{6}(t)$ is almost zero. Instead, for $V_{O}>300$ the systems exhibit very pronounced Bragg peaks [large values of $I(t)$ ] and an the order parameter $\Psi_{6}(t)$ is large. Therefore, we refer to those systems as ordered systems. 

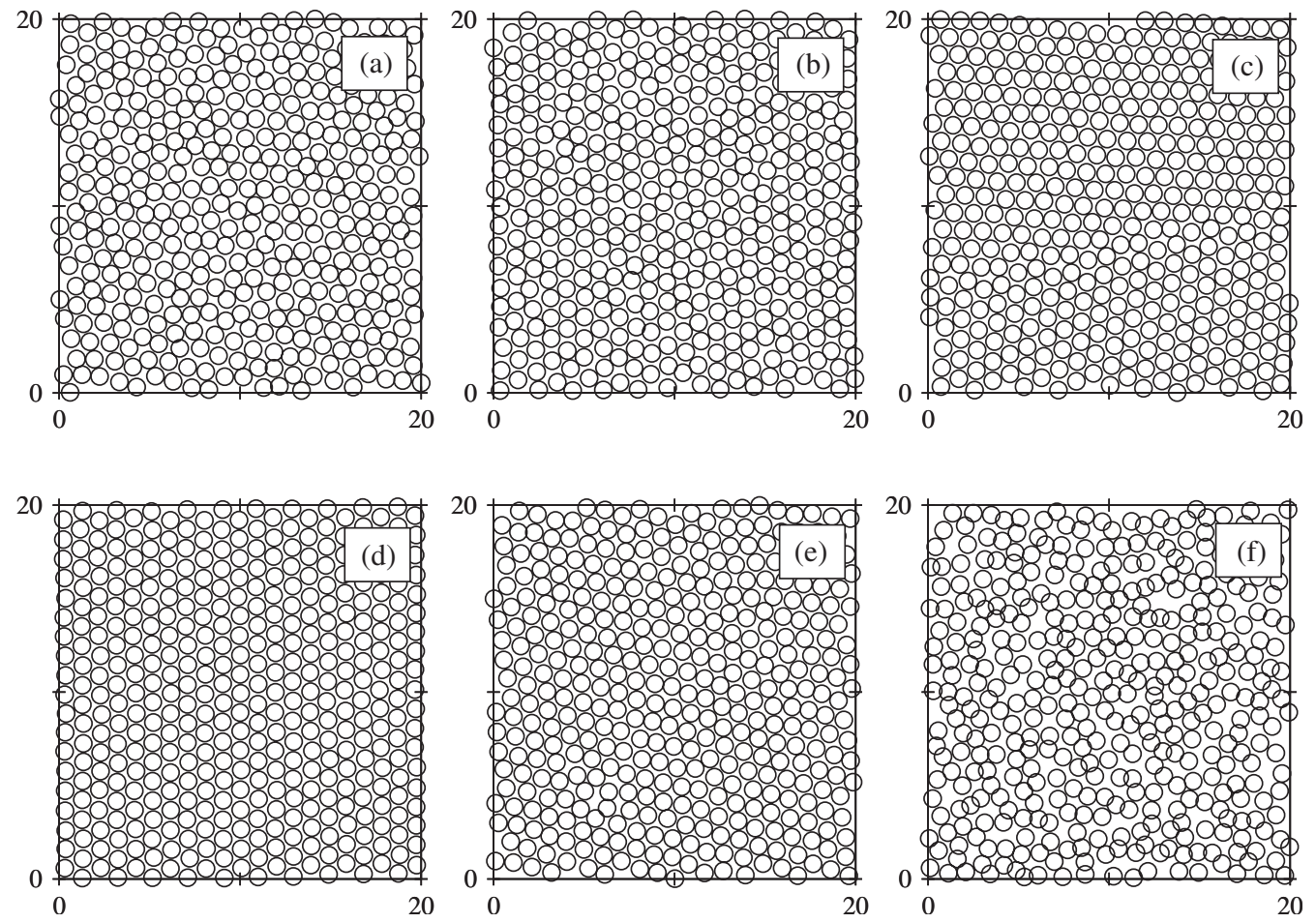

FIG. 1. Snapshots of conformations in the steady state both in the absence and presence of shear. For the sake of clarity, only about $1 / 10$ th of the system is shown. The particle number density is chosen to be the same for all snapshots. (a), (b), and (c) correspond to zero shear rates and $V_{O}=250,300,2000$, respectively, and illustrate the order-disorder transition observed at zero shear rate, $\dot{\gamma}=0$. Notice in (c) a defect boundary that indicates the existence of at least two different domains (polycrystalline system). Snapshots (d), (e), and (f) correspond to $V_{O}=2000$ and shear rates $\dot{\gamma}=0.001,0.2,0.4$, respectively. A maximum degree of order is observed in snapshot (d) clearly indicating the existence of an optimal shear rate for maximum ordering.

\section{B. Results for dense charge stabilized colloids}

Figure 1 shows several snapshots of final conformations for different values of $V_{O}=250,300,2000$ both in the presence and absence of shear flow. For the sake of clarity only about $1 / 10$ th of the system area is shown. Snapshots (a), (b) and (c) illustrate the order-disorder transition observed at zero shear, $\dot{\gamma}=0$. Snapshot (c) shows the existence of polycrystalline domains even at $V_{O}=2000$. In Fig. 1, snapshots (d), (e), and (f) show typical steady state conformations for $V_{O}=2000$ and for three different shear rates $\dot{\gamma}=10^{-3}, 0.2$, and 0.4 respectively. A comparison among snapshots (c), (d), (e), and (f) points out the existence of an optimum shear rate for which the ordering is maximum [snapshot $(d)]$. A quantitative analysis is performed by measuring the temporal evolution of $\Psi_{n}(t)$ and $I(t)$ (Fig. 2). A transition order-disorder occurs around $V_{O}^{c} \sim 250-300$, which is roughly the same value at which the transition was observed for $3 \mathrm{D}$ systems in the simulations performed by Butler-Harrowell [38]. Above $V_{O}^{c}$ the system exhibits typical features of a highly correlated system and the degree of ordering increases with $V_{O}$. Whereas below $V_{O}^{c}$ the system remains in low ordered conformations similar to liquid-gas states, for $V_{O}>V_{O}^{c}$ the analysis of the different orientation parameter orders $\Psi_{n}(t)$ shows that the system adopts conformations close to a HCP crystal, and $\Psi_{6}(t)$ is the most relevant quantity to follow the temporal evolution of the degree of order in the system. The inset in Fig. 2 shows the evolution of $\Psi_{6}(t)$ with time for different values of $V_{O}$. Results are similar to the ones observed using $I(t)$. It should be noticed that the degree of ordering grows with time but tends to saturate at long times. This final plateau indicates that the system has reached a stationary state

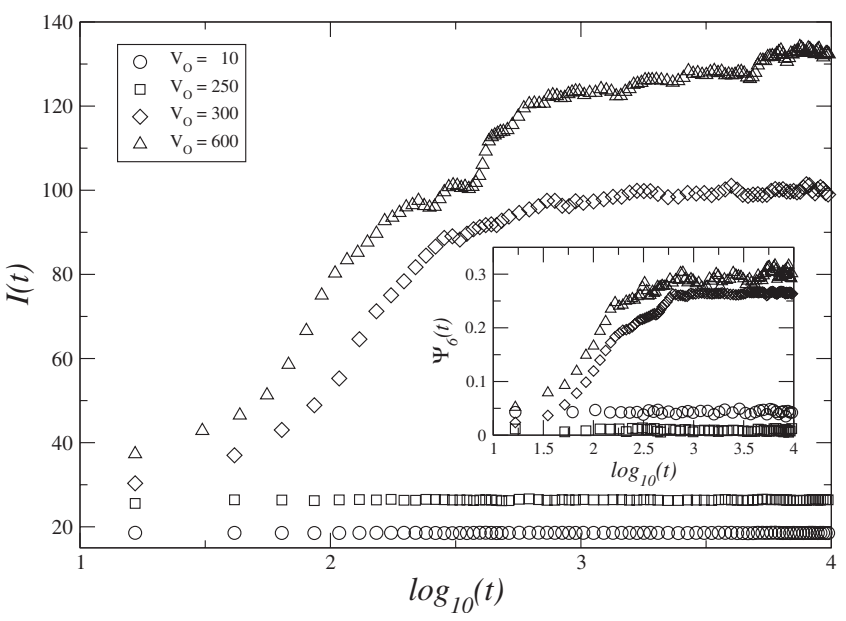

FIG. 2. Temporal evolution of $I(t)$ (observable related to the degree of order in the sample, see text) in nonsheared systems for different strength of the Yukawa potential $V_{O}$. Order is characterized here using the measure $I(t)$, see definition in the text. A transition from a disordered to an ordered system is observed at $V_{O}^{c}$ $\sim 250-300$. The inset shows temporal evolution of order using the orientation order parameter $\Psi_{6}(t)$. 

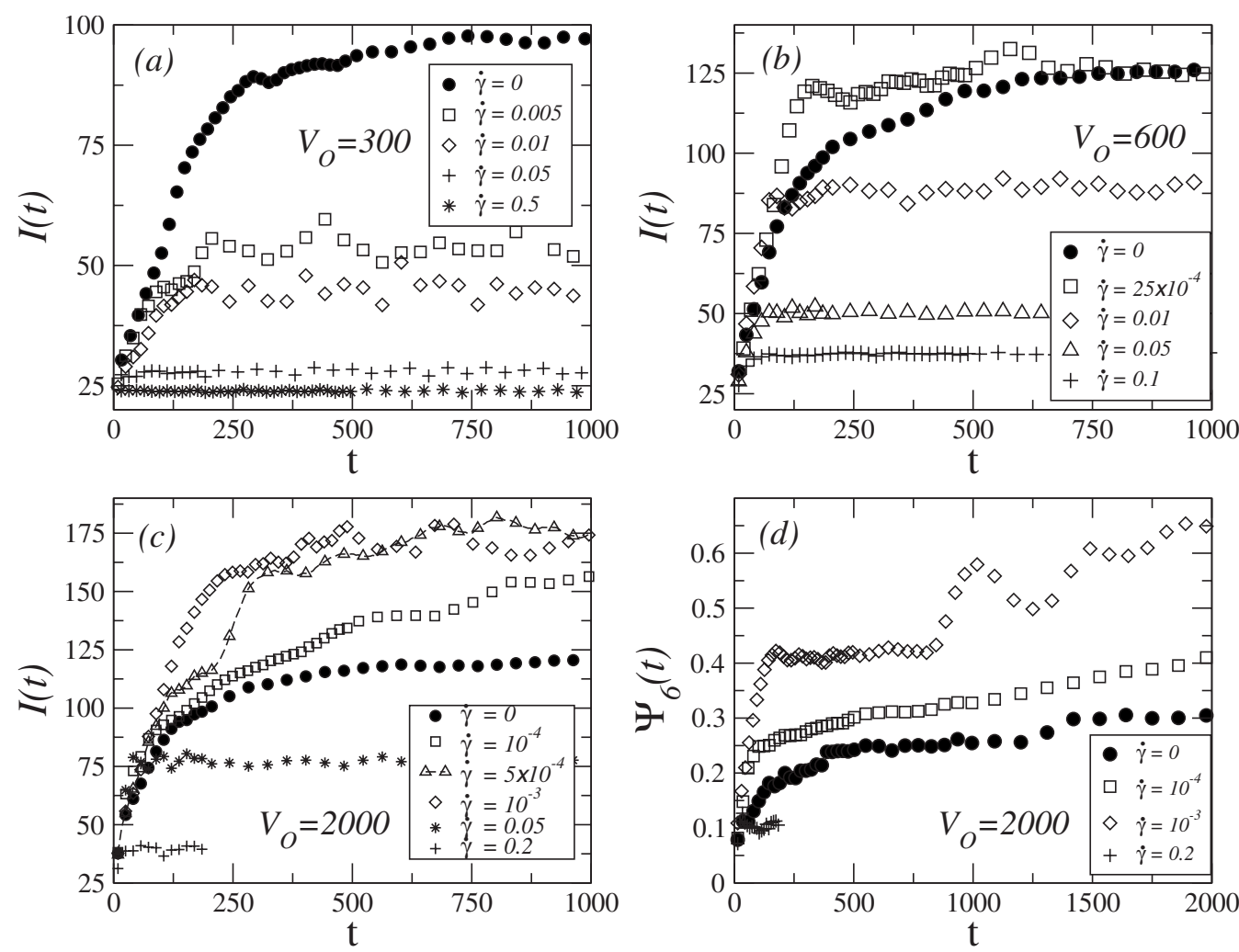

FIG. 3. Temporal evolution of the degree of order for different values of $V_{O}$ and shear rates $\dot{\gamma}$. The plots (a), (b), and (c) correspond to the evolution of the order parameter $I(t)$ for $V_{O}=300,600,2000$, respectively. (d) shows the evolution of the orientation order parameter $\Psi_{6}(t)$ for $V_{O}=2000$. Approximated Deborah numbers for these systems can be obtained using the rule De $\sim \dot{\gamma} / 10$ (see text).

in which the averaged observables do not further evolve in time. The ordering increases with $V_{O}$ although perfect ordering of the system is not achieved even for $V_{O}$ much larger than $V_{O}^{c}$ probably due to the formation of polycrystalline domains as observed in Fig. 1 snapshot (c). It should be noticed that the obtaining of a perfect order could be hampered by the square geometry of the simulation box used in this work, which cannot fit a perfect triangular lattice. Although, as Fig. 1(d) shows, the square geometry of the simulation box does not prevent the formation of monodomains with particles in positions slightly distorted from a perfect triangular lattice.

The kinetics of the order-disorder transition changes when the system is sheared. Figure 3 shows the temporal evolution of the degree of order in the system using $I(t)$ [plots (a), (b), (c)] and the orientation order parameter $\Psi_{6}(t)[$ plot $(d)]$ for $V_{O}=300,600,2000$. Results show that when $V_{O}$ is increased low shear rates help to speed-up the order-disorder transition, and for an optimal shear rate a larger degree of crystallinity is observed when compared to nonsheared systems (solid circles). These effects can be understood in terms of the dual role that shear plays on the ordering of the system. On the one hand, low shear rates can help to break the diffusion barrier between different crystal domains, remove boundaries, and merge them into larger crystals. This favorable effect of shear is expected to be more noticeable if Brownian diffusion is small compared to the diffusion induced by shear, i.e., at large values of the potential parameter $V_{O}$. On the other hand, high shear rates distort and finally destroy large crystals due to the imposed mechanical stress. This negative effect of shear is more important for low values of $V_{O}$ as softer potentials would lead to a smaller effective exclude volume and, as a consequence, the entropic force that maintains the crystal cohesion is reduced and cannot effectively oppose to the mechanical stress imposed by shear.

It should be also noted in Fig. 3 that by increasing the shear rate beyond the optimal values, shear effectively destroys the transition from disordered to ordered states as observed previously in the work of $\mathrm{BH}$. In their work, $\mathrm{BH}$ tested systems at $V_{O} \sim 600$ with Deborah numbers larger than De $\gtrsim 0.01$, which roughly corresponds to $\dot{\gamma} \gtrsim 0.1$ in our simulations. A clear agreement is observed for those shear rates between $\mathrm{BH}$ and our results. The evolution of the disorder-order transition can be also measured using the order parameter $\Psi_{6}(t)$. Plot (d) in Fig. 3 shows the temporal evolution of $\Psi_{6}(t)$ for several shear rates at $V_{O}=2000$. Results are similar to the ones obtained using $I(t)$, although the system continues with arrangements to further increase the degree of order. Note also that the rate of ordering at zero shear rates is much smaller than those for the optimal shear rates. Thus, low shear rates provide a clear advantage of promoting a higher degree of crystallization in a short period of time whereas higher shear rates hamper the ordering of the system. These results are consistent with the experimental work of Holmqvist et al. [31] where the crystal growth rate for a given concentration of charged colloids was found to exhibit a maximum as a function of shear rate. Remarkably, Mokshin-Barrat [39], in their recent work with shifted 


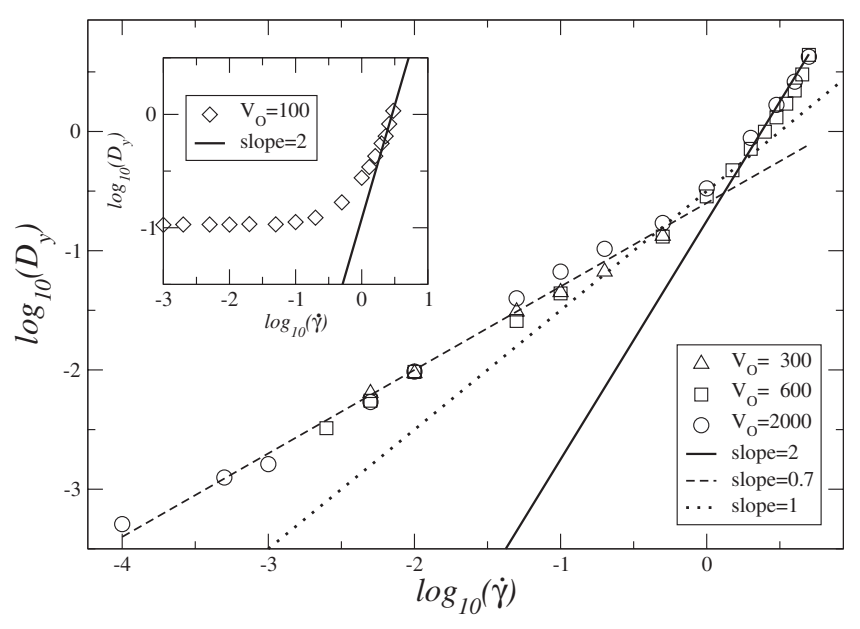

FIG. 4. Diffusion coefficient along the gradient direction $D_{y}$ as a function of the shear rate in a log-log plot. Dashed, dotted, and solid lines with slopes $0.7,1$, and 2 , respectively, are introduced to provide guidance to the eye. Results are observed to agree with the form predicted by Breedveld and Levine for low to moderate shear rates but not for large shear rates. The inset shows the diffusion coefficient $D_{y}$ for $V_{0}=100$ (a system that does not show a transition to an ordered phase, see Fig. 2).

Lennard-Jones particles, have also reported small amounts of shear to enhance the degree of order in the system.

Analysis of the behavior of the diffusion coefficient along the gradient direction $D_{y}$ allows us a further characterization of these sheared systems. The diffusion coefficient $D_{y}$ is computed via the mean-squared displacement of the particles as

$$
\left\langle\left[y(t)-y\left(t_{o}\right)\right]^{2}\right\rangle_{\text {traj }}=2 D_{y}\left(t-t_{o}\right),
$$

where $\langle\cdots\rangle_{\text {traj }}$ stands for the average over the different trajectories. Figure 4 shows the dependence of the diffusion coefficient $D_{y}$ on the shear rate $\dot{\gamma}$. In all our simulations, shear is observed to enhance diffusion although we have observed previously that the crystalline order in the systems is enhanced at low shear rates. At low shear rates, a regime with power law $D_{y} \sim \dot{\gamma}^{0.7}$ is observed for $V_{O}>V_{O}^{c}$. On the other hand, at larger shear rates a different power-law regime with $D_{y} \sim \dot{\gamma}^{2}$ is observed. The two regimes observed in the numerical simulations can be compared with the predictions of a theoretical model similar to the one used by BreedveldLevine (BL) [61]. Here, the original three-dimensional BL model has been straightforwardly adapted to the twodimensional geometry of our problem. The theoretical model does not allow us to predict absolute values for the diffusion coefficient $D_{y}$, but a quick estimation of the behavior of $D_{y}$ as a function of $\dot{\gamma}$ is possible. Our analysis shows that the BL model predicts at low shear rates a regime $D_{y} \sim \dot{\gamma}^{0.7}$ in both two and three dimensions. This prediction is in remarkable agreement with the behavior observed in Fig. 4. When shear is gradually increased in the theoretical model, a short plateau followed by a slight decrease of $D_{y}$ occurs before the onset to a different power-law regime given by $D_{y} \sim \dot{\gamma}$. In our simulations (Fig. 4) no plateau is observed and $D_{y} \sim \dot{\gamma}^{2}$ for larger shear rates. Although the nature of the mismatch between the model and simulations at large shear rates is unknown, we note that in simulations the crossover regime roughly occurs in the range of shear rates at which the solid phase melts away. At high shear rates the diffusion is expected to be basically the diffusion of the disordered fluid. The inset of Fig. 4 shows the behavior of $D_{y}$ with the shear rate for $V_{O}=100$. In those systems in which solid phase is absent independently of the applied shear rate, a power-law $\dot{\gamma}^{2}$ similar to the case $V_{O}>V_{O}^{c}$ is observed for shear rates large enough. As expected in all the systems, in the limit $\dot{\gamma}$ $\rightarrow 0$, an asymptotic decay towards the zero shear diffusion constant $D_{y}\left(\dot{\gamma}=0, V_{O}\right)$ is observed. When $V_{O}$ increases, the free mobility of the particles is reduced, and for $V_{O}>V_{O}^{c}$ the decay to the zero shear diffusion constant occurs at shear rates much lower that those depicted in Fig. 4. This asymptotic plateau when $\dot{\gamma} \rightarrow 0$ should not to be confused with the plateau predicted by the BL model at moderate shear rates.

\section{DEPLETION-DRIVEN COLLOIDS}

The aim of this section is the study of attractive colloids, namely, those which pair potential has an attractive well which depth is no larger than a few $k_{B} T$ 's. In order to study this kind of colloidal system we have chosen as a representative example, the Asakura-Oosawa-Vrij (AOV) potential [41] which has proved to be useful to mimic 3D polymerdepletion driven colloidal systems when the volume fraction of colloids is low enough. We should remark that our aim is not to study the specific case of three-dimensional polymer depletion-driven systems via the Asakura-Oosawa-Vrij approach, but use AOV potential to obtain conclusions that might be representative to the common behavior of colloidal systems interacting via shallow short-ranged attractive potentials at the same time that it allows us to link easily our work to previous colloidal studies using the same kind of potential [62-64].

\section{A. System description}

A pair-interaction potential $U$ consisting of two contributions is assumed: The Asakura-Oosawa-Vrij potential [41] $\left(U_{\mathrm{AO}}\right)$ plus a repulsive inverse power interaction $\left(U_{h c}\right)$ given by the following expressions:

$$
U\left(r_{i j}\right)=U_{\mathrm{AO}}\left(r_{i j}\right)+U_{h c}\left(r_{i j}\right),
$$

$$
\begin{aligned}
& \frac{U_{\mathrm{AO}}\left(r_{i j}\right)}{k_{B} T} \\
& = \begin{cases}\frac{3 \phi_{P}}{2 \zeta^{3}}\left((1+\zeta)^{2} r_{i j}-\frac{r_{i j}^{3}}{3}-\frac{2(1+\zeta)^{3}}{3}\right) & \text { for } r_{i j}<(1+\zeta), \\
0 & \text { for } r_{i j}>(1+\zeta),\end{cases}
\end{aligned}
$$

$$
\frac{U_{h c}\left(r_{i j}\right)}{k_{B} T}=r_{i j}^{-n},
$$

where $r_{i j}=\left|\mathbf{r}_{i}-\mathbf{r}_{j}\right|$ is the relative distance between particles. The total pair potential $U=U_{\mathrm{AO}}+U_{h c}$ passes through a mini- 
TABLE I. Relation between nonadsorbing polymer volume fraction, $\phi_{p}$ and the absolute value $\left|U_{m}\right|$ of the potential minimum for $\zeta=0.1$.

\begin{tabular}{lc}
\hline \hline$\phi_{p}$ & $\left|U_{m}\right| / k_{B} T$ \\
\hline 0.2293 & 3.2 \\
0.2334 & 3.3 \\
0.2414 & 3.5 \\
0.3664 & 7.0 \\
0.5998 & 15.0 \\
\hline \hline
\end{tabular}

mum value $\left(U_{m}\right)$ which is related to the parameter $\phi_{P}$. Thus, the parameters $\zeta$ and $\phi_{P}$ control the scope and depth of the attractive well created by the depletion-driven interaction (see Table I). In our simulations $\zeta=0.1$ as in previous work $[63,64]$. Thus the interaction is quite short ranged and is cutoff at a reduced distance of $1.1 \sigma_{c}$, where $\sigma_{c}=\sigma$ is the monomer diameter. For completeness, it should be recalled that in the original context of polymer-depletion driven colloidal systems, the parameter $\zeta$ would correspond to the sizeratio between a polymer chain and a colloidal particle, while $\phi_{P}$ is the polymer volume fraction. In the inverse power repulsive interaction given by Eq. (11), the exponent has been set to $n=36$. Exponents $n<36$ are reported [66] to lead to anomalies when a hard-core mimic is required in the potential. The area fraction of particles is set to $f_{v} \approx 0.157$. In sheared systems, in order to ensure independence of the results, with respect to the box size, preliminary simulations using different length boxes $L=128,256,512,1024$ have been initially performed at different shear rates. It has been observed that for $L \geqslant 256$, results are independent of the box size. Therefore the usual length of the system is set to $L$ $=256$ which corresponds to $N_{p}=13107$ particles in the system. Nonetheless, in several cases, for deep quenches and low shear rates, simulations of length $L=512,1024$ have been used in order to ensure accurate statistics at large times. Time steps range from $d t=10^{-3}$ at zero shear to $d t=10^{-4}$ for $\dot{\gamma}=1$.

At zero shear rate, the transition from a single dispersedphase to a two-phase coexistence is observed in the present model when the system is quenched to $U_{m}<U_{c}$ being, $U_{c}$ $\approx-3.130 k_{B} T$. In that case, a phase separation process starts and homogeneous cluster nucleation is observed. At late times, large round shaped clusters coexist with a sea of monomers and some small aggregates [64]. Colloidal particles are arranged in a hexagonal packing inside such clusters. By increasing $\left|U_{m}\right|$ further, i.e., deep into the two-phase region, fractal clusters are obtained at late times [64]. Here, the interfacial responsible for the surface reorganization of monomers is almost frozen and the cluster shape results mainly from random cluster-cluster collisions as in a traditional diffusion-limited cluster-cluster aggregation (DLCA) or reaction-limited cluster-cluster aggregation (RLCA) models. However, even for this well depth, the aggregates show hexagonal closed-packed crystalline ordering at short length scales while displaying a ramified fractal nature at larger length scales. In the present work several quenches into the two phase region are studied, $\left|U_{m}\right|=3.2,3.3,3.5$ (shallow

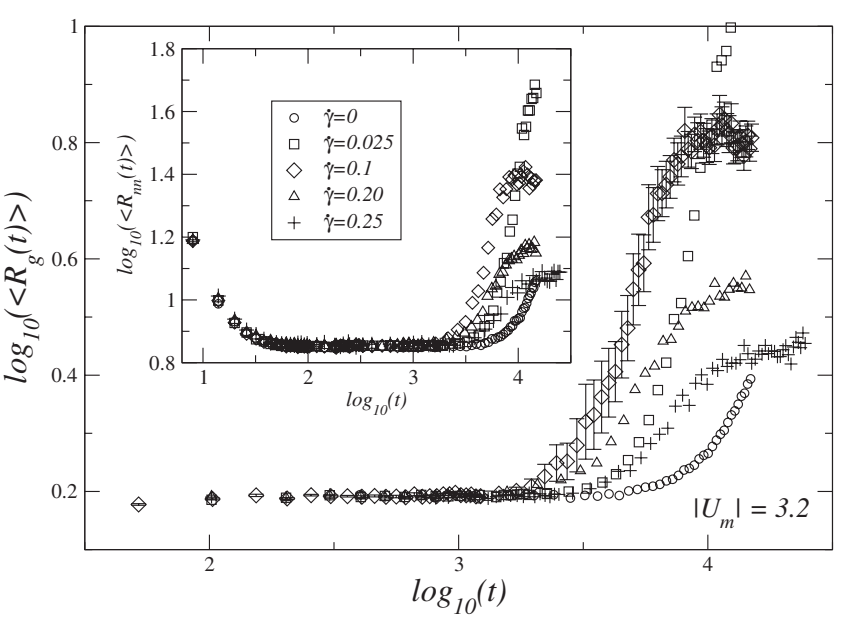

FIG. 5. Temporal evolution of the mean cluster radius of gyration $\left\langle R_{g}(t)\right\rangle$ for different shear rates in polymer-depletion driven colloids quenched to $\left|U_{m}\right|=3.2$ close to the critical value $U_{c}$ $\approx 3.130 k_{B} T$ where the order-disorder transition occurs. Error bars using confidence intervals of $90 \%$ (student's $t$-distribution) are depicted for shear rate $\dot{\gamma}=0.1$ as a representative example of the magnitude of the errors involved in the measures obtained by averaging over runs with different initial conditions. The inset plot shows the behavior of the mean distance between clusters $\left\langle R_{n n}(t)\right\rangle$ for the same systems. In both cases, a plateau is observed at initial times $100<t<2000$ which indicates the existence of an initial induction time previous to the formation of viable nuclei for crystallization. Note how both $\left\langle R_{n n}(t)\right\rangle$ and $\left\langle R_{g}(t)\right\rangle$ show a remarkable similarity in their temporal evolution over this time period.

quenches) and $\left|U_{m}\right|=7,15$ (deep quenches). These values of $\left|U_{m}\right|$ are selected to be representative examples of systems displaying compact and fractal clusters at zero shear rate.

In defining clusters, we consider that a particle is linked to another one if the distance between them $\left|\mathbf{r}_{i j}\right|<r_{\text {cut }} \equiv(1+\zeta)$. We calculate the radius of gyration of the clusters, $R_{g}$, and cluster-cluster nearest-neighbor separation $R_{n n}$. In general, only clusters larger than $N=5$ particles are considered for the calculation of the averaged values $\left\langle R_{g}(t)\right\rangle$ and $\left\langle R_{n n}(t)\right\rangle$. For each time $t$, statistical averages over 20 different runs have been performed for each quench.

\section{B. Role of shear at initial stages of the cluster nucleation}

\section{Analysis of the induction time}

In the absence of shear it has been observed [64] that after quenching the system into the two-phase region $\left(\left|U_{m}\right|\right.$ $\left.\geqslant\left|U_{c}\right|\right)$ there is an initial period of time, known as induction time when very small clusters or nuclei try to grow but eventually revert to single particles [65]. The induction time is expected to depend on both the quench depth $\left|U_{m}-U_{c}\right|$ and shear rate. Figure 5 shows the temporal evolution of the mean cluster radius of gyration $\left\langle R_{g}(t)\right\rangle$ for a shallow quench $\left|U_{m}\right|=3.2$ where it shows a plateau after only a few time steps. During this period, small clusters do not evolve into larger ones. The same behavior is observed In the inset of 


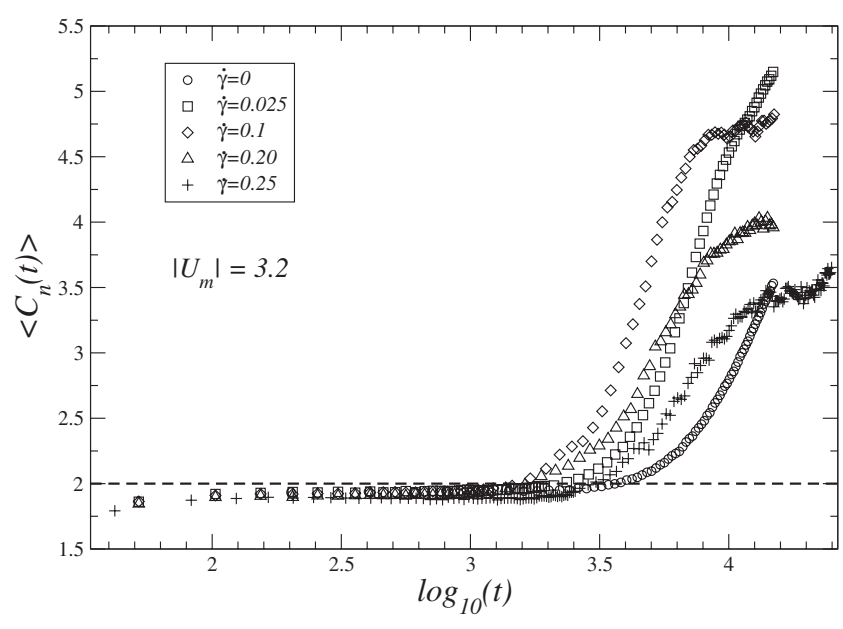

FIG. 6. Temporal evolution of the mean coordination number $\left\langle C_{n}\right\rangle$ for different shear rates considered in Fig. 5.

Fig. 5 for the mean cluster-cluster nearest-neighbor separation $\left\langle R_{n n}(t)\right\rangle$. Three different regimes can be distinguished in Fig. 5: An initial preplateau regime in which the systems start forming initial aggregation seeds $\left[\left\langle R_{g}(t)\right\rangle\right.$ grows, and the distance between clusters $\left\langle R_{n n}(t)\right\rangle$ decreases, see [67] for more details]; a second stage [the first plateau, $t$ $\in(100,2000)]$ in which seeds try to grow in size but thermal fluctuations break the seeds before they can reach the critical size to have a sustained growth; and finally a third stage in which due to the randomness of the aggregation phenomena, some nuclei manage to exceed the critical size before fading away, and the crystallization process starts. It should be noticed that data in Fig. 5 has been averaged over 20 runs, and therefore individual cluster size fluctuations cannot be observed in Fig. 5. At large times, the onset to a second plateau is observed in Fig. 5. This second plateau corresponds to the steady state regime, and is discussed later in the paper.

In order to estimate the value of the induction time, the mean coordination number $\left\langle C_{n}(t)\right\rangle$ is computed. This is defined as the mean number of links per particle for particles with at least one link. Thus, $\left\langle C_{n}(t)\right\rangle=1$ indicates that the system is composed only of monomers and dimers. Figure 6 shows the coordination number for several shear rates with $\left|U_{m}\right|=3.2$. The plateau regime is reached in a few time steps and the mean coordination time stays roughly steady below $\left\langle C_{n}(t)\right\rangle=2$. The plateau period ends with a sudden increase of $\left\langle C_{n}(t)\right\rangle$. A practical definition of the induction time $\tau_{i}$ is obtained by defining it as the time the system needs to reach the value $\left\langle C_{n}(t)\right\rangle=2$ which points to the time at which the plateau ends.

The induction times computed for different shear rates are depicted in Figs. 7(a) and 7(b). Results show that induction times reduce substantially as one moves slightly away from the order-disorder phase boundary $\left(U_{c} \approx-3.130 k_{B} T\right)$. Nonetheless, in shallow quenches $\left|U_{m}\right|=3.2,3.3$ an optimal shear rate that minimizes the induction time is observed. Beyond that point, shear is detrimental to the ordering of the system.

Thus, close to the phase boundary, small amounts of shear help to trigger the aggregation process. These results agree remarkably with the experimental results of Penkova et al. [28] where they find that very small shear rates strongly affect nucleation. The authors also show the existence in some systems of an optimal shear rate that corresponds to a maximum nucleation rate. Enhancement of crystallization by shearing seen in our simulations is also in agreement with other recent experimental studies where stirring the solution is reported to enhance the crystallization process $[29,30]$. The existence of an optimal shear rate could be of crucial importance in initializing the crystallization of many substances. Furthermore, as Penkova et al. suggested in their work, the existence of an optimal shear rate could also help to explain the differences observed between crystallization experiments carried out in microgravity and earth-based laboratories.

Both the induction times as well as the range of shears beneficial to crystallization shrink for deeper quench depths $\left(\left|U_{m}\right| \gg\left|U_{c}\right|\right)$. Remarkably, the induction times grow asymptotically to $\infty$ at a finite shear rate $\dot{\gamma}^{*}\left(U_{m}\right)$ that depends on the

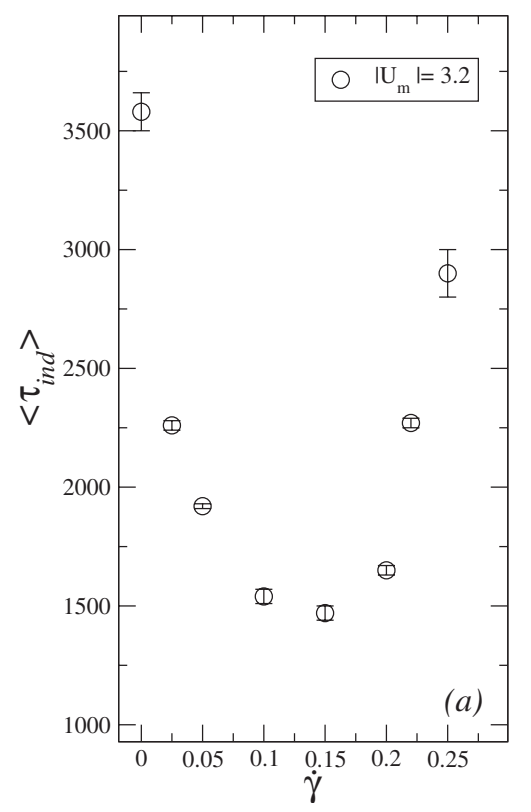

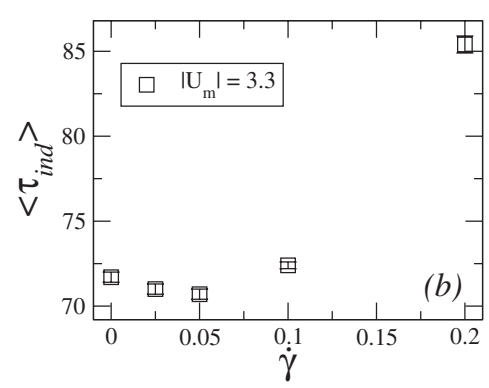

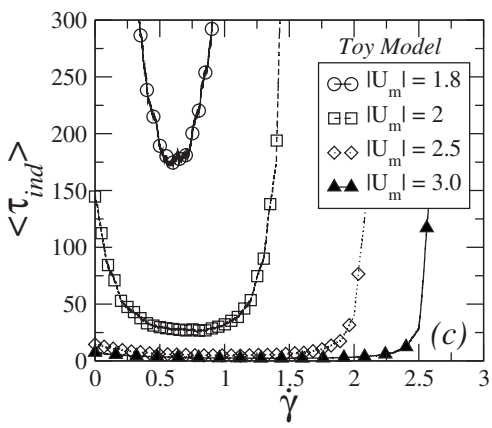

FIG. 7. (a) and (b) show the induction time as a function of shear rate for quenches $\left|U_{m}\right|$ $=3.2,3.3$, respectively. Results clearly show that for systems close to the order-disorder transition boundary $U_{c} \approx 3.130 k_{B} T$, there is an optimal shear rate that minimizes the induction time before the start of the crystallization. Plot (c) depicts the induction times predicted by a toy model (see text) using the following model parameters: Critical size $n_{t h}=10, l_{b}=1, B_{S}=B_{B}=1$, and $B_{e}$ $=1000$ (see text). Modification of these parameters changes the absolute values obtained with the toy model but the qualitative trends are similar to those obtained in (a) and (b). 


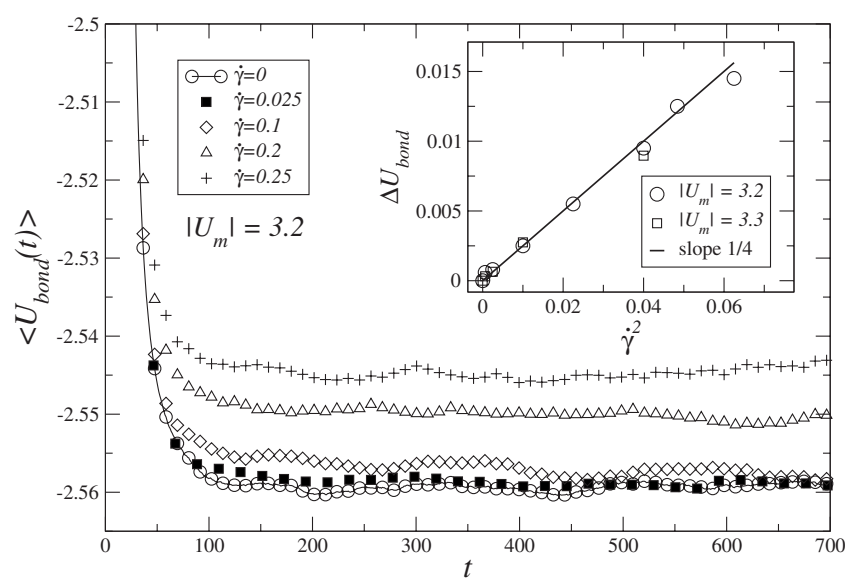

FIG. 8. Mean interaction energy $\left\langle U_{\text {bond }}(t)\right\rangle$ between pairs of particles inside clusters are shown for several shear rates during the induction time in systems quenched to $\left|U_{m}\right|=3.2$. The mechanical stress created by the shear tends to increase the distance between particles and the interaction between two particles is less binding. The inset shows a comparison between the energy shifts $\Delta U_{\text {bond }}$ $\equiv U_{\text {bond }}(\dot{\gamma})-U_{\text {bond }}(\dot{\gamma}=0)$ predicted by Eq. (12) and the simulations for quenches $\left|U_{m}\right|=3.2,3.3$. The solid line is the theoretical prediction, circles and squares are values for $\left|U_{m}\right|=3.2$ and $\left|U_{m}\right|=3.3$, respectively.

quench depth. This implies that for $\dot{\gamma}>\dot{\gamma}^{*}$ crystallization is suppressed because no viable nuclei are able to form [68].

\section{Dual nature of shear}

An important question that needs to be addressed now is why low shear rates trigger the nucleation process. Shear is expected to weaken the effective attractive interaction between neighboring particles due to the mechanical stress that pulls apart particles which have slightly different relative positions along the gradient direction. Indeed, this negative effect of shear is observed in our simulations. Figure 8 shows how shear weakens the effective particle-particle interaction between neighboring particles $\left\langle U_{\text {bond }}(t)\right\rangle$ during the induction period. In the measurement of $\left\langle U_{\text {bond }}(t)\right\rangle$, two particles are considered to form a pair (bond or link) if its relative distance $r_{i j} \leqslant 1+\zeta$. When the shear rate is zero, $\left\langle U_{\text {bond }}(t)\right\rangle$ is expected to be close to the minimum $U_{m}$ but shifted towards more positive values due to the thermal fluctuations and the asymmetry of the potential. Indeed, the observed shifts are quite small, but close to the order-disorder transition boundary, any subtle change in the effective interaction can be extremely important. A rough estimation of the shift in the effective energy induced by shear is possible. Here the focus is on the case of small clusters (most of them dimers) found during the induction period. The calculation of the shift in the effective energy for larger clusters as those found in the stationary regime is more involved and will be discussed later in Sec. IV C 2.

In order to explain the changes of $\left\langle U_{\text {bond }}(t)\right\rangle$ during the induction period, it is enough to consider the slight difference in velocities $\Delta v=\dot{\gamma} \Delta y$ that exist between two bonded particles having slightly different positions along the gradient direction. For the sake of simplicity particles are assumed to be separated by a constant distance $l_{b}$. A further assumption is that all bond orientations have the same probability to occur (the last condition will hold when thermal noise is much larger than the anisotropy imposed by shear). Thus, the excess kinetic energy between two linked particles due to translation is

$$
\Delta K_{t} \equiv\left\langle\frac{m}{2}\left(\Delta v_{i j}\right)^{2}\right\rangle=\frac{m}{4} l_{b}^{2}(\dot{\gamma})^{2},
$$

where particle mass $m$ is taken as unity, and $\langle\cdots\rangle$ implies an average over all possible relative orientations. The kinetic energy due to rotations for small clusters is neglected. It should be pointed out that the inverse will hold for large clusters. The difference between the value of $\left\langle U_{\text {bond }}(t)\right\rangle$ at a certain shear rate $\dot{\gamma}$ and the value at zero shear rate must be roughly equal to difference in kinetic energies, i.e., $\Delta K_{t}$ $\approx U_{\text {bond }}(\dot{\gamma})-U_{\text {bond }}(\dot{\gamma}=0)$. The inset of Fig. 8 shows a remarkable agreement between predictions from Eq. (12) and simulations for shallow quenches $\left|U_{m}\right|=3.2,3.3$. during the induction time.

Thus, the observation of an optimal nonzero shear rate for the induction time implies that a second effect must counteract the negative effects of shear on crystallization. As it has been suggested in previous works $[31,39]$, a plausible explanation is that shear enhances the rate of collisions between particles with respect to Brownian diffusion which in turn will enhance the probability of creating a viable nucleus. A hint pointing in this direction can be obtained from the following simplified toy model in two dimensions. Let $f_{c}(n)$ be the mean rate of single particles colliding against a cluster of size $n$, which is a function of the radius of the cluster $r(n)$ $\propto n^{1 / 2}$. The collisions are due to both Brownian movement, and shear. The part of the collision rate due to the Brownian movement of the particles is expected to be proportional to the section $r(n)$ of the cluster. On the other hand the collision rate due to shear is expected to be proportional to $f_{v} \dot{\gamma} r^{2}(n)$. The last can be proved easily by assuming the cluster to be at $y=0$ and exposed to a flow of particles with a velocity $v_{x}$ $=\dot{\gamma} y$. The number of particles the cluster collects during a $d t$ interval in the section $(y, y+d y)$ is $d N_{(y, y+d y)}=f_{v}\left|v_{x}\right| d t d y$ $=f_{v}|y| \dot{\gamma} d t d y$. Integrating $d N_{(y, y+d y)} / d t$ over all section segments, i.e., from $-r(n)$ to $+r(n)$ leads to the aforementioned result. Therefore, the total collision rate can be written as

$$
f_{c}(n) \equiv B_{S} r^{2}(n) \dot{\gamma}+B_{B} r(n),
$$

where the factors $B_{S}$ and $B_{B}$ are adjustable parameters corresponding to shear and Brownian rates, respectively. Once a particle collides with the cluster of size $n$, it has a certain probability of aggregating that is proportional to the number of bonds the particle can establish with the neighbor particles in the surface of the cluster $n_{b}$. Each bond will contribute to the Boltzmann factor of the bonded state with an energy $U_{\text {bond }}(\dot{\gamma}=0)+\Delta K$ where $\Delta K$ is given in Eq. (12). The energy associated to the nonbonded state is taken as zero. Therefore, the aggregation probability of a colliding particle is 


$$
P\left[r(n), n_{b}\right]=\frac{\exp \left\{-n_{b}\left[U_{\text {bond }}(\dot{\gamma}=0)+\Delta K\right]\right\}}{1+\exp \left\{-n_{b}\left[U_{\text {bond }}(\dot{\gamma}=0)+\Delta K\right]\right\}},
$$

where for simplicity $n_{b}=n$ for clusters of size $n \leqslant 3$, and $n_{b}$ $=4$ for $n \geqslant 4$. Values of $n_{b}>4$ are not considered because new aggregating particles will stick to the periphery of the cluster and therefore they will be only partially surrounded by other particles of the cluster. In fact, during nucleation, clusters are not expected to be very large, and as a further simplification it can be assumed that all particles in the cluster are proposed to leave the cluster with the same rate $B_{e}$. The particle escape is accepted with probability $P_{e}\left(r_{n}, n_{b}\right)$ $=1-P\left(r_{n}, n_{b}\right)$. The qualitative behavior of the induction times can be assessed using the toy model by measuring the time a cluster of size $n=1$ needs to grow in size up to a certain threshold size $n_{t h}$ which represents the critical size for a cluster to be large enough to sustain a continuous growth against thermal fluctuations. Plot (c) in Fig. 7 shows the induction times obtained from the toy model to reach a critical size $n_{t h}=10$ with parameters $l_{b}=1, B_{S}=B_{B}=1$ and $B_{e}$ $=1000$. A comparison of plots (a) and (b) with (c) in Fig. 7 shows that the behavior of the induction times in the toy model have qualitatively the same trends: Optimal shear rate, reduction of the induction times when deeper quenches are performed, and an asymptotic behavior of the induction time at high shear rates. The qualitative predictions of the toy model are unaffected by reasonable changes in the value of the parameters of the model $B_{S}, B_{B}, B_{e}, n_{t h}$. Thus, despite its simplicity, the toy model shows that shear can play a positive role in nucleation due to the enhanced transport it promotes. The optimal shear rate would arise from the competition of the two opposing effects of shear: Weakening of the effective pair interaction and enhancement of particle transport.

\section{Steady state}

\section{Characterization of the mean cluster size}

Once the induction period ends, nuclei of the solid phase (crystal) starts to grow into large clusters. Under shear the system is expected to reach a steady state with a sheardependent mean cluster size [26,43,46,48,51] (see Fig. 5). In addition, previous simulations have reported shear to promote the compaction of fractal clusters [69]. This is consistent with our observations for deep quenches (see Fig. 9 for $\left.\left|U_{m}\right|=15\right)$. The degree of compactness is observed to increase with the shear rate. As for deep quenched systems with low shear rates the mean size of the clusters is very large in the steady state; we often needed to increase the system size to $L=512$ and $L=1024$. The temporal evolution of sheared systems depicted in Fig. 9 seems to suggest that in the low shear regime clusters aggregate until some critical size is reached when shear tends to break the big clusters. Note also the increased number of monomers that coexist with large aggregates at high shear rates.

An estimation [70] of the mean critical size of clusters $N>50$ when they break $\left\langle s_{\text {break }}\right\rangle_{\text {steady }}$ as a function of the shear rate and the quench $\left|U_{m}\right|$ is presented in Table II. The steady state mean values, denoted by $\langle\cdots\rangle_{\text {steady }}$ have been obtained by averaging over 20 different runs the conformations in the steady state. A comparison of $\left\langle s_{\text {break }}\right\rangle_{\text {steady }}$ with the mean size of the clusters larger than $N=5$ particles $\langle s\rangle_{\text {steady }}$ shows that in general the breaking size is $30 \%-40 \%$ larger than the mean size. Therefore, from the inspection of Table II and Fig. 9 it can be concluded that the existence in the steady state of a perpetual cycle in which clusters aggregate among them until a certain critical size is exceeded and the metastable cluster breaks mainly into several smaller pieces that restart the process of aggregation.

Despite the fact that clusters seem to break under the influence of shear at sizes larger than the mean size of the clusters in the system, the mean values are still expected to be representative of the steady states in sheared systems. Figure 10 shows the mean radius of gyration $\left\langle R_{g}\right\rangle_{\text {steady }}$ of clusters larger than $N=5$ particles in the steady state as a function of the shear rate. At low shear rates a power-law regime $\left\langle R_{g}\right\rangle_{\text {steady }} \propto \dot{\gamma}^{-1 / 3}$ is observed for shallow and moderate quenches $\left|U_{m}\right|=3.5,7$. For deep quenches $\left|U_{m}\right|=15$ the exponent seems to be larger than $-1 / 3$, but smaller than an exponent $-1 / 2$. Doi and Chen [46] using a two-dimensional sticky particle model, reported an exponent $-2 / 3$ for simulations corresponding to very deep quenches. The exponent seen in our study is not that large, however, this does not rule out that the value of the exponent increases with the value of the quench $\left|U_{m}\right|$ towards the Doi-Chen reported exponent. Remarkably, the $-1 / 3$ power law has been already predicted by Wessel and Ball [44] who derived it by assuming that the bending moment induced by shear, $R F \sim R^{3} \xi \sim R^{3} \eta_{o} \dot{\gamma}$ (where $\xi$ is the shear stress), needs to exceed a certain critical value that depends on the pair energy in order to break the cluster. We would also like to point out that an exponent value of $-1 / 3$ is also seen in phase separating polymer mixtures under shear [71-73].

At high shear rates, a larger exponent characterizing a reduction in the size of clusters beyond the predicted $\gamma^{-1 / 3}$ behavior is observed in Fig. 10. A possible explanation for this further reduction in size is the existence of parallel mechanisms (different from cluster breaking), like surface erosion of small particles that increases in importance for high shear rates. The inset of Fig. 10 shows the fraction $\chi$ of particles in clusters of size $N \leqslant 5$ for several quenches $\left|U_{m}\right|$ $=3.5,7,15$ : A clear correlation between the breakdown of the power law $\dot{\gamma}^{-1 / 3}$ and the presence of clusters $N<5$ is observed. Thus, beyond a certain shear rate not only the breaking of oversized clusters contributes to limit the growth of clusters but also the continuous erosion of particles becomes an important mechanism. The fragmentation and erosion processes in two-dimensional aggregates have been recently reviewed by Vassileva et al. [74]. Their observation that erosion occurs only beyond a critical shear rate close to the shear rate necessary for fragmentation is consistent with our simulation results.

In addition, Fig. 10 shows that the breakdown of the $\gamma^{-1 / 3}$ regime depends on the depth of the quench $\left|U_{m}\right|$. This result is consistent with the idea that at constant shear rate erosion is less probable to occur for strongly attractive particles. It should be noted that the erosion mechanism is in part balanced by an extra flow of eroded particles arriving at the cluster from other clusters. In this way the net effect of shear consists of shifting partially the dynamical equilibrium be- 

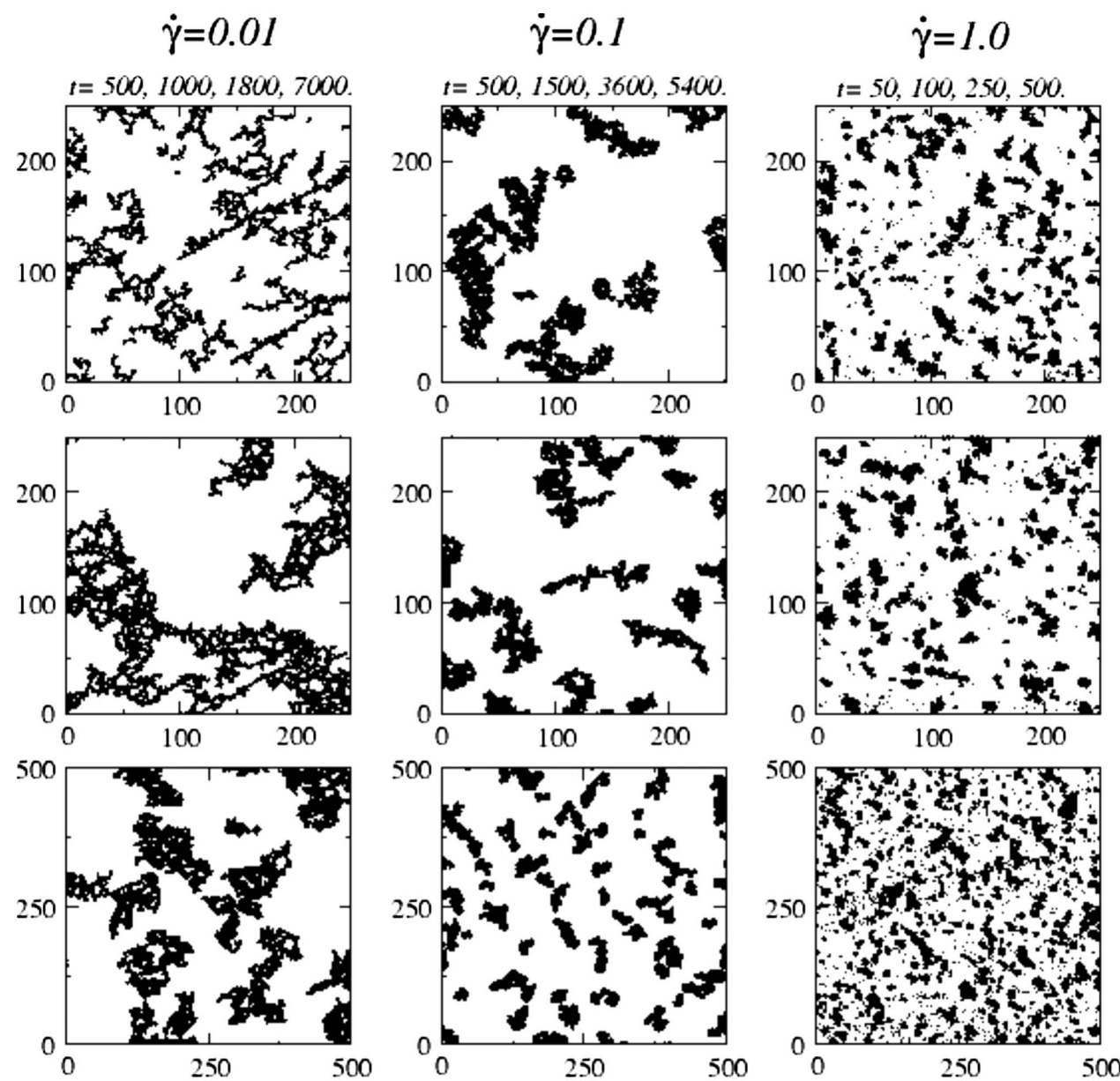

FIG. 9. Snapshots showing the temporal evolution of a deep quenched system, $\left|U_{m}\right|=15$, for three different shear rates $\dot{\gamma}$ $=0.01,0.1,1.0$. For the sake of clarity, only a fraction of the total area of the simulated systems is shown. Shear is observed to compact the initial fractal aggregates, and break large clusters into smaller pieces. For high shear rates, an additional mechanism consisting of the erosion of single and small aggregates from the surface of large clusters also becomes important. Last frames correspond to typical snapshots already in the steady state regime (at higher shear rates the dynamics is faster and smaller times are needed to attend the steady state).
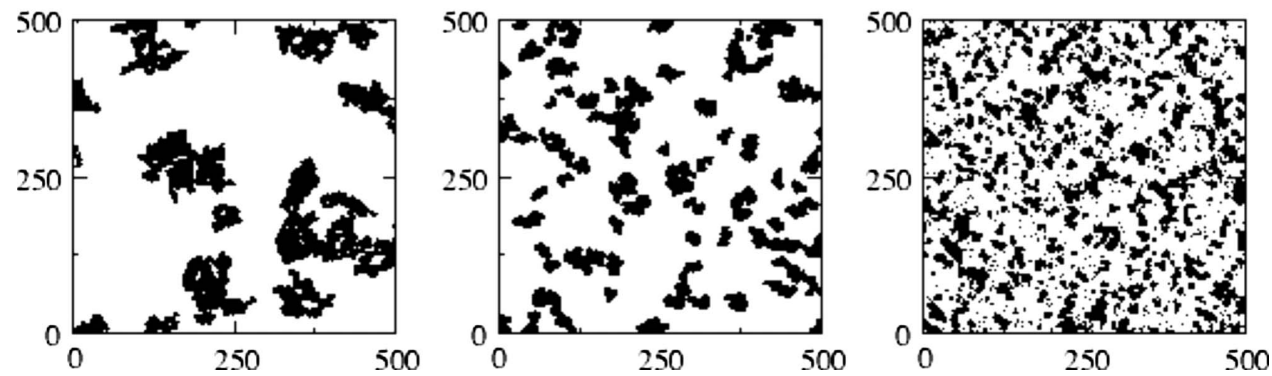

tween the free particles and large clusters towards the formation of small clusters.

\section{Importance of the rotation of the clusters}

The combination of shear and friction forces acting on the cluster leads to the rotation of the aggregates with respect to the perpendicular axis passing along their center of mass. The effect induced by the rotation of a cluster as a rigid body increase in importance with the size of the cluster. Rotations are expected to decrease the energy per bond and make the clusters less stable. Effect of rotations in the stability of bonds between particles have been invoked by Selomulya et al. [51] in order to explain their experimental results in which particles reach a maximum size before reaching a significantly smaller steady state value. The inset (a) of Fig. 11 shows the measured mean angular velocities of the clusters $\langle\omega\rangle_{\text {steady }}$ as a function of the shear rate $\dot{\gamma}$ for $\left|U_{m}\right|=3.5,15$. In the case of circular clusters it is straightforward to show that

the angular velocity of a cluster behaves as $\omega=\dot{\gamma} / 2$. Results in inset (a) show a remarkable agreement between the mean angular velocities obtained in simulations and the theoretical prediction $\dot{\gamma} / 2$ (dashed line). The mean angular velocities can be used to evaluate the total kinetic energy of circular clusters due to rotation $M_{c} R_{g}^{2}\langle\omega\rangle^{2} / 2$ where $M_{c}$ is the mass of the cluster. The rotation plus the translation of the cluster as a rigid body stretches the bonds between particles, and therefore the effective energy per bond is expected to be weaker than at zero shear rate. The increase in the energy per bond can be easily estimated if particles inside the circular cluster are assumed to be packed forming a hexagonal compact lattice and edge effects are neglected. In that case the number of effective bonds per particle is three and the kinetic energy per bond due to rotations is $K_{r}=m R_{g}^{2} \dot{\gamma}^{2} / 24$. Thus, combining translational [see Eq. (12)] and rotational energies, the increase in energy per bond is expected to be 
TABLE II. Results obtained in the steady state regime for the mean size of clusters larger than $N=50$ when they break $\left\langle s_{\text {break }}\right\rangle_{\text {steady. The mean size of clusters larger than } N=5 \text { particles }}$ $\langle s\rangle_{\text {steady }}$, and the estimated value of the mass break according to Eq. (18) $\left\langle S_{\text {break }}^{\text {(model) }}\right\rangle$ are also shown.

\begin{tabular}{|c|c|c|c|c|}
\hline$\left|U_{m}\right| / k_{B} T$ & $\dot{\gamma}$ & $\left\langle s_{\text {break }}\right\rangle_{\text {steady }}$ & $\langle s\rangle_{\text {steady }}$ & $\left\langle S_{\text {break }}^{(\text {model })}\right\rangle$ \\
\hline \multirow[t]{9}{*}{3.5} & 0.01 & 2470 & 1700 & 9950 \\
\hline & 0.016 & 2190 & 1350 & 5580 \\
\hline & 0.025 & 2050 & 1500 & 3220 \\
\hline & 0.04 & 1230 & 1000 & 1800 \\
\hline & 0.06 & 840 & 615 & 1100 \\
\hline & 0.1 & 560 & 400 & 580 \\
\hline & 0.16 & 380 & 235 & 320 \\
\hline & 0.4 & 145 & 32 & 90 \\
\hline & 0.6 & 43 & 10 & 48 \\
\hline 7 & 0.1 & 730 & 560 & 900 \\
\hline \multirow[t]{9}{*}{15} & 0.01 & 6200 & 4900 & 19000 \\
\hline & 0.016 & 4410 & 3270 & 13700 \\
\hline & 0.025 & 2990 & 2300 & 7850 \\
\hline & 0.04 & 2290 & 1670 & 4420 \\
\hline & 0.1 & 1140 & 880 & 1430 \\
\hline & 0.16 & 950 & 710 & 800 \\
\hline & 0.4 & 480 & 310 & 250 \\
\hline & 0.6 & 330 & 210 & 140 \\
\hline & 1.0 & 200 & 100 & 70 \\
\hline
\end{tabular}

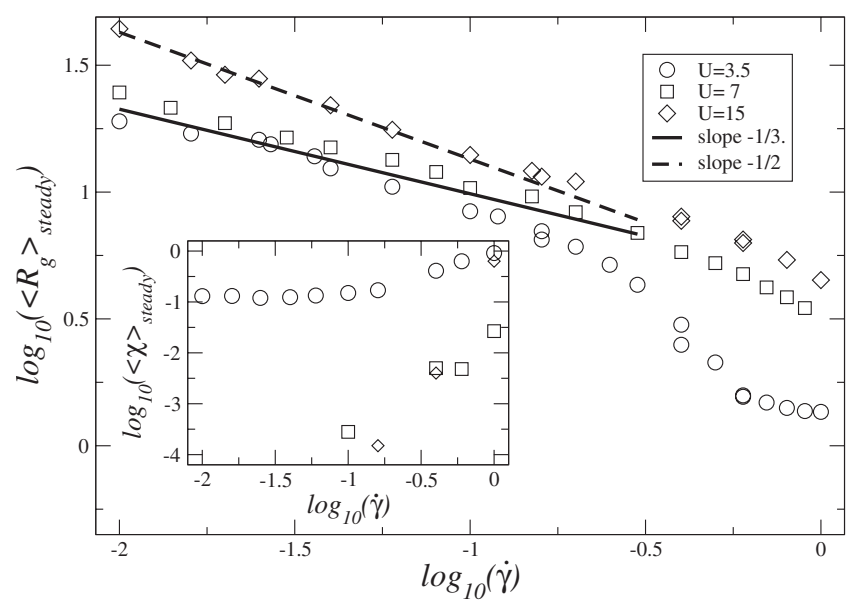

FIG. 10. The mean radius of gyration $\left\langle R_{g}\right\rangle_{\text {steady }}$ of clusters larger than $N=5$ in the steady state is plotted as a function of the shear rate in a log-log plot. To guide the eye, lines with slope $-1 / 3$ and $-1 / 2$ have been plotted. For quenches $\left|U_{m}\right|=3.5,7$ a $\dot{\gamma}^{-1 / 3}$ power-law regime is observed at low shear rates, for deep quenches $\left|U_{m}\right|=15$ the exponent is larger than $-1 / 3$ but lower than $-1 / 2$ (see text). The inset shows in a log-log scale the average fraction $\langle\chi\rangle_{\text {steady }}$ of clusters with size $N \leqslant 5$ for different quenches $\left|U_{m}\right|=3.5,7,15$. A correlation between the breakdown of the $\dot{\gamma}^{-1 / 3}$ law and the increase of the number of single and small clusters $N \leqslant 5$ is observed.

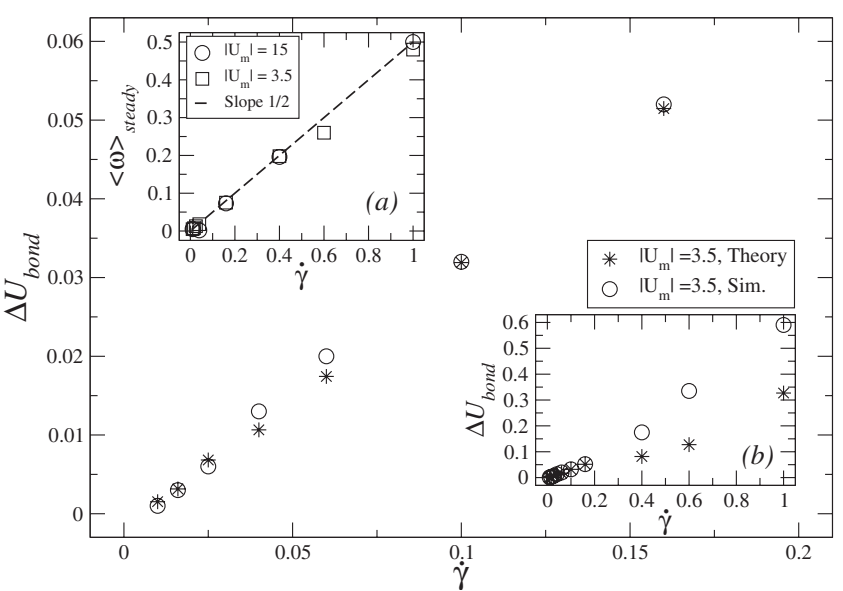

FIG. 11. The change in the effective energy per bond in the steady state $\Delta U_{\text {bond }}=U_{\text {bond }}(\dot{\gamma})-U_{\text {bond }}(\dot{\gamma}=0)$ is shown as a function of the shear rate $\dot{\gamma}$ for $|U|=3.5$. Circles denote simulation results and stars denote our theoretical prediction. Mean radii of gyration $\left\langle R_{g}\right\rangle_{\text {steady }}$ obtained from simulations are used to evaluate Eq. (15). The inset plot (a) shows the mean angular velocity of the clusters in the steady state regime $\langle\omega\rangle_{\text {steady }}$ as a function of shear rate for $\left|U_{m}\right|=3.5,15$. The dashed line shows the theoretical prediction $\langle\omega\rangle_{\text {steady }}=\dot{\gamma} / 2$. The inset plot (b) depicts the same as the main figure but for a larger range of shear rates.

$$
\begin{aligned}
\Delta U_{\text {bond }} & \equiv U_{\text {bond }}(\dot{\gamma})-U_{\text {bond }}(\dot{\gamma}=0) \\
& =K_{r}+K_{t}=\frac{m}{24} R_{g}^{2} \dot{\gamma}^{2}+\frac{m}{4} l_{b}^{2} \dot{\gamma}^{2} .
\end{aligned}
$$

Figure 11 and the inset (b) show a comparison between the theoretical prediction for $\Delta U_{\text {bond }}$ (stars) and the simulation results (open circles) for $\left|U_{m}\right|=3.5$. A remarkable agreement is observed for low shear rates whereas roughly a factor 2 of difference is observed at high shear rates [high shear rates data are shown in the inset (b)]. The mismatch at high shear rates can be explained due to the reduction in size of the clusters and the subsequent increase of the ratio between the particles in the periphery of the cluster and the inner particles forming a hexagonal compact structure. Under these circumstances the number of effective bonds per pair is expected to reduce from three to lower values when shear is increased, and therefore $\Delta U_{\text {bond }}$ is expected to be larger than Eq. (15) predicts.

The remarkable agreement between the simulation results and the predictions for circular rigid bodies can be used to attempt to introduce a simple model in order to explain the cluster breaking. In this model, the kinetic energy gained by a cluster is assumed to be eventually responsible for the breaking of the cluster. It is simple to show that the total kinetic energy of a circular aggregate, once the velocity of the center of mass has been discounted, can be approximated (ignoring edge effects) as

$$
K_{T}(N)=m \dot{\gamma}^{2}\left(q \frac{1}{16} N^{2} r_{p}^{2}+\frac{3}{4} N l_{b}^{2}\right),
$$

where $r_{p}$ is the radius of a monomer, and $q$ is the packing factor for a $2 \mathrm{D}$ hexagonal lattice which is $\pi / \sqrt{12} \approx 0.9$. The relations $M_{c}=N m$ and $R_{g}^{2}=q r_{p}^{2} N / 2$ have been used. 
It is easy to show using Eq. (16) that large circular clusters are in a metastable state with respect to various splitting modes. For instance, in the case of binary splitting $N$ $\rightarrow N / 2+N / 2$, if clusters are large enough that kinetic energy is the dominant contribution, then the energy would roughly reduce by a factor of one-half if splitting is allowed $K_{T}(N)$ $>2 K_{T}(N / 2) \sim K_{T}(N) / 2$. Nonetheless the splitting reaction involves a barrier energy roughly equal to the number of bonds that need to be broken along one of the diameters of the circle $E_{\text {barrier }}(N) \approx 3 U_{\text {bond }}(\dot{\gamma}) \sqrt{q N}$. The probability of a cluster of size $N$ to cross the potential barrier $P_{\text {break }}$ can be modeled as

$$
P_{\text {break }} \sim \exp \left(\frac{-E_{\text {barrier }}(N)}{K_{T}(N)}\right) .
$$

Once the breaking probability is known, the mean breaking size can be estimated as

$$
\left\langle S_{\text {break }}^{(\text {model })}\right\rangle=\int_{0}^{\infty} f(N) N d N,
$$

where $f(N)$ represents the probability density function of clusters able to grow up to size $N-1$ but that split at size $N$, i.e.,

$$
f(N) \equiv \frac{g(N)}{\sum_{i=1}^{\infty} g(i)},
$$

where the function $g(x)$ is defined as

$$
g(x) \equiv\left(\prod_{i=1}^{x-1}\left[1-P_{\text {break }}(i)\right]\right) P_{\text {break }}(x) .
$$

The last column in Table II shows the predicted mean breaking sizes using Eq. (18) for the corresponding systems. In that calculation $r_{p}=0.5, U_{\text {bond }} \rightarrow\left|U_{m}\right|, l_{b}=1$. Despite many simplifications used in the model, the values predicted by Eq. (18) are of the same order of magnitude when compared to the mean breaking sizes observed in simulations. In fact, for shallow quenches and large shear rates, in which clusters resemble circular aggregates, the agreement is remarkable.

The observed key role of the kinetic energy in the stability of the clusters suggests that systems having a similar relation between the kinetic energy of clusters measured in bond units should be closely related. A simple calculation shows that the total kinetic energy associated with a circular cluster can be related to its mean radius of gyration as $K$ $\sim \gamma^{2}\left(\left\langle R_{g}\right\rangle_{\text {steady }}\right)^{4}$. Figure 12 shows that plotting the mean kinetic energy of clusters $K$ in units of bond energy $U_{\text {bond }}$ $\rightarrow\left|U_{m}\right|$ as a function of the product of the mass of the cluster $M_{c}$ and the shear rate $\dot{\gamma}$ leads to a master curve for our data. Figure 12 in addition to simulations with friction constant $\Gamma=0.5$ also contain results from additional simulations performed at $\Gamma=2$. The inset plot shows that for quenches $|U|$ $=3.5,7$ the data roughly follows a power law with exponent $2 / 3$.

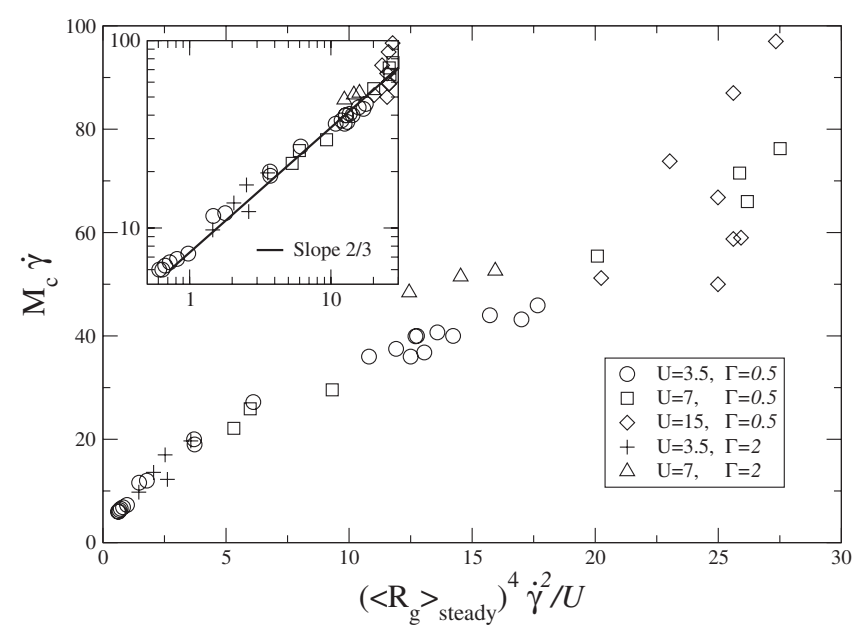

FIG. 12. The figure shows that simulation data can be mapped onto a master curve by plotting the kinetic energy of the cluster in units of pair interactions $\left\langle R_{g}\right\rangle_{\text {steady }}^{4} \dot{\gamma}^{2} / U$ versus the product of the shear rate and the mean number of monomers (mass) inside the clusters $\dot{\gamma} M_{c}$. Here, two different friction constants $\Gamma=0.5,2$ have been used. The inset plot is a log-log plot of the same data in which data roughly fit a power law with exponent $2 / 3$.

\section{CONCLUSIONS}

Extensive Langevin dynamics simulations have been used in this work to study the effects of shear rates on the kinetics of crystallization in colloidal systems. Two different systems such as charge stabilized (Yukawa repulsive) and depletion driven attractive (Asakura-Oosawa) colloids have been chosen as model colloidal systems. Results show that in both cases, low shear rates can promote an enhancement of the kinetics of crystallization.

Low shear rates are observed to speed up the ordering of the system. For Yukawa repulsive colloids low shear plays an important role when the strength of the potential $V_{O}$ is large. At optimal shear rates the degree of order is larger than the one obtained for zero shear. This seems to arise from the fact that shear helps to iron out defects that otherwise makes the sample polycrystalline. For larger shear rates, similar to the ones previously studied by Butler and Harrowell [17] in their 3D simulations, our results confirm that shear substantially hampers crystallization. Similarities between our 2D model and the 3D results of $\mathrm{BH}$ at zero shear rate and at moderate Deborah numbers suggest that the existence of an optimal shear rate seen in our 2D simulations should also be present in 3D. Such a 3D simulation of the order-disorder process is currently under study.

Analysis of the diffusion coefficient along the gradient direction is carried out. We find that the diffusion coefficient increases with a power law with the shear rate as $D_{y} \sim \dot{\gamma}^{0.7}$ followed by a crossover to a regime $D_{y} \sim \dot{\gamma}^{2}$. The exponent 0.7 is in agreement with the predictions of the BreedveldLevine [61] theoretical model adapted for our twodimensional geometry.

In the case of depletion-driven attractive colloids, our results show that low shear rates can reduce the induction time needed for crystals to grow. This effect is strong close to the 
phase boundary of the order-disorder transition. An optimal shear rate that minimizes the induction time is observed in the simulation. This can be explained in terms of the competition of two opposite effects of shear: On the one hand, shear is observed to weaken the effective interaction between particles due to the mechanical stress, but on the other hand shear provides an extra flow of particles to the nuclei which can speed up their growth in addition to the usual flow of particles provided by Brownian movements. This analysis is supported by a simple theoretical model in which predicted induction times show similar characteristics to the results observed in our simulations. Use of low shear flows could have important implications in crystallization processes (of proteins, for example) allowing the speed up of the crystallization process and yielding higher quality crystals.

Enhancements of the aggregation kinetics similar to what we observed in our simulations has been previously reported in several experiments [28-31], and in the very recent work of Mokshin-Barrat [39]. The present work also helps to put into context previous simulation works $[38,37]$ that concluded that shear had a negative effect on aggregation. Such claims are correct for the range of shear rates covered in the previous simulations. However, as mentioned earlier, enhancing effects of shear can be observed at lower shear rates. We also note that very high shear rates could induce a reentering transition to the ordered state as observed experimentally by Haw et al. [33].

Shear is observed to induce a steady state in which the aggregates (crystals) reach a limiting mean size. In the case of deep quenches, shear compress the initial fractal clusters. At low shear rates the aggregation process continues until a critical size is reached when the cluster is not able to maintain the cohesion due to the mechanical stress and ends up being split in to a few pieces which restart the aggregation process. In this regime it is possible (at least for shallow and moderate quenches) to characterize the dependence of the mean cluster size as a function of the shear rate via a $\left\langle R_{g}\right\rangle_{\text {steady }} \propto \dot{\gamma}^{-1 / 3}$ power law previously predicted [44]. At higher shear rates it is speculated that the erosion of particles from the cluster surface also plays an important role in limiting the cluster size in the steady state. This leads to a deviation of the $\left\langle R_{g}\right\rangle_{\text {steady }} \propto \dot{\gamma}^{-1 / 3}$ power law in favor of a faster decrease of $\left\langle R_{g}\right\rangle_{\text {steady }}$ with the shear rate $\dot{\gamma}$.

A detailed analysis of the cluster rotations induced by shear shows that the rotational kinetic energy of clusters is important in determining the stability of large clusters. A very rudimentary model based on considering the kinetic energy of circular aggregates have been introduced. The model is observed to roughly predict the mean size of the breaking clusters for those cases in which clusters resemble circular aggregates. Most of the simulation systems for depletion driven colloids are observed to collapse onto a master curve when the kinetic energy of the cluster expressed in bond units is plotted against the product of the cluster mass and the shear.

The present work has been performed in the NVT ensemble. For constant pressure simulations (Gibbs ensemble), we expect the main qualitative trends to hold. In the case of repulsive colloids at constant pressure, we expect the particles to have an effective exclude volume smaller than in the case the volume of the system is fixed. Thus, the transition to the ordered state as well as the beneficial effects of little amounts of shear on crystallization are expected to be shifted to higher repulsive strengths of the potential (higher $V_{O}$ ). In the case of attractive colloidal systems at constant pressure, due to the low density of colloidal particles, their aggregation is expected to have a minor contribution to the change of the pressure in the system, and therefore the only important parameter to characterize the behavior of the system is the area fraction of colloids present in the system. The area fraction can be either regulated via pressure or by fixing the volume of the system to a certain size. We expect that our results would stimulate further theoretical and experimental studies towards the understanding of the role of shear for colloidal systems in widely different physical situations.

\section{ACKNOWLEDGMENTS}

C.H. and J.C. thank the DFG via Grants TR6 and Ho1108/ 16-1 for partial financial support. . T.S. thanks Ministerio de Educación y Ciencia (Spain) Contract No. FIS07-60327. C.S. and A.C. are supported by NSF-NIRT Grant No. 0609318.

\section{APPENDIX}

In this study, simulations using boundary conditions similar to Lees-Edwards (LE) boundary conditions [60] are performed. For a flow profile as given in Eq. (2), the original LE boundary conditions state that if a particle leaves the system in the gradient direction $\pm \hat{e}_{y}(y \in[0, L])$, it will be reinserted in the simulation box with a velocity in the shear direction $\hat{e}_{x}$ corrected by $\mp V_{d} \hat{e}_{x}$, where $V_{d} \equiv \dot{\gamma} L$ is the difference in velocities between the two sides of the cell in the $\hat{e}_{y}$ direction. The boundary conditions for the positions can be summarized as

$$
\begin{gathered}
a=\operatorname{NINT}\left(\frac{y^{(\text {old })}}{L}-\frac{1}{2}\right), \\
b=x^{\text {(old) }}-a t V_{d}, \\
x^{(\text {new })}=b-L \operatorname{NINT}\left(\frac{b}{L}-\frac{1}{2}\right), \\
y^{(\text {new })}=y^{\text {(old })}-a L,
\end{gathered}
$$

where NINT $[h]$ is the nearest integer to $h$. The nearest image convention using Lees-Edwards boundary conditions can be summarized as

$$
\begin{gathered}
\Delta x^{*}=x_{i}-x_{j}, \\
\Delta y^{*}=y_{i}-y_{j}, \\
c=\operatorname{NINT}\left(\frac{\Delta y^{*}}{L}\right),
\end{gathered}
$$




$$
\begin{gathered}
d=\Delta x^{*}-c t V_{d}, \\
\Delta x=d-L \operatorname{NINT}\left(\frac{d}{L}\right), \\
\Delta y=\Delta y^{*}-c L .
\end{gathered}
$$

In our work, the van Gunsteren-Berendsen algorithm (BDGB) [57] is used to perform the simulations. This algorithm uses only positions at steps $t$ and $t-d t$ to compute positions at $t+d t$. Thus, the Lees-Edwards boundary condi- tions should be modified in the context of BDGB algorithm in the following way: Once the new position has been computed and it is detected that a particle is crossing the boundaries in the direction $\pm \hat{e}_{y}$, we apply the usual corrections and before the beginning of a new step, the change $x(t)$ $\rightarrow x(t) \mp V_{d} d t$ is performed. Although this is a simplified picture, we do obtain the desired mean velocity profiles and confirm that the fluctuating part of the velocity profiles [cf. Eq. (2)] do not depend of the distance of the particles from the boundaries when proper time steps $d t$ are used in the simulations as mentioned in Secs. III and IV.
[1] J. D. Joannopoulos, P. R. Villeneuve, and S. Fan, Nature (London) 386, 143 (1997).

[2] G. Pan, R. Kesavamoorthy, and S. A. Asher, Phys. Rev. Lett. 78, 3860 (1997).

[3] J. H. Holtz and S. A. Asher, Nature (London) 389, 829 (1997).

[4] S. Pechenov, B. Shenoy, M. X. Yang, S. K. Basu, and A. L. Margolin, J. Controlled Release 96, 149 (2004).

[5] L. J. DeLucas and C. E. Bugg, Trends Biotechnol. 5, 188 (1987).

[6] C. Lawrence, M. E. Fabry, and R. L. Nagel, Blood 78, 2104 (1991).

[7] C. R. Berland, G. M. Thurston, and M. Kondo, Proc. Natl. Acad. Sci. U.S.A. 89, 1214 (1992).

[8] A. Ponce, C. Sorensen, and L. Takemoto, Mol. Vis 12, 879 (2006).

[9] N. Enomoto, T. Sung, Z. Nakagawa, and S. Lee, J. Mater. Sci. 27, 5239 (1992).

[10] P. G. Vekilov, B. R. Thomas, and F. Rosenberger, J. Phys. Chem. B 102, 5208 (1998).

[11] L. DeLucas, K. Moore, M. Long, R. Rouleau, T. Bray, W. Crysel, and L. Weise, J. Cryst. Growth 237, 1646 (2002).

[12] A. K. Arora and B. V. R. Tata, Adv. Colloid Interface Sci. 78, 49 (1998).

[13] A. Yethiraj, Soft Matter 3, 1099 (2007).

[14] J. Vermant, Curr. Opin. Colloid Interface Sci. 6, 489 (2001).

[15] P. Butler, Curr. Opin. Colloid Interface Sci. 4, 214 (1999).

[16] A. Onuki, J. Phys.: Condens. Matter 9, 6119 (1997).

[17] S. Butler and P. Harrowell, J. Chem. Phys. 103, 4653 (1995), and references therein.

[18] J. Delhommelle, Phys. Rev. B 69, 144117 (2004), and references therein.

[19] M. J. Stevens and M. O. Robbins, Phys. Rev. E 48, 3778 (1993), and references therein.

[20] S. E. Paulin, B. J. Ackerson, and M. S. Wolfe, J. Colloid Interface Sci. 178, 251 (1996).

[21] P. A. Thomson, and M. O. Robbins, Science 250, 792 (1990).

[22] R. Messina and H. Löwen, Phys. Rev. E 73, 011405 (2006), and references therein.

[23] A. Stipp, R. Biehl, T. Preis, J. Liu, A. Barreira, H. J. Schöpe, and T. Palberg, J. Phys.: Condens. Matter 16, S3885 (2004).

[24] A. Tsuchida, E. Takyo, K. Taguchi, and T. Okubo, Colloid Polym. Sci. 282, 1105 (2004).

[25] R. M. Amos, J. G. Rarity, P. R. Tapster, T. J. Shepherd, and S.
C. Kitson, Phys. Rev. E 61, 2929 (2000).

[26] J. Vermant and M. J. Solomon, J. Phys.: Condens. Matter 17, R187 (2005).

[27] J. K. G. Dhont, Phys. Rev. Lett. 76, 4269 (1996).

[28] A. Penkova, W. Pan, F. Hodjaoglu, and P. G. Vekilov, Ann. N.Y. Acad. Sci. 1077, 214 (2006), and references therein.

[29] D. Li and R. B. Kaner, J. Mater. Chem. 17, 2279 (2007); J. Am. Chem. Soc. 128, 968 (2006).

[30] P. Jiang and M. McFarland, J. Am. Chem. Soc. 126, 13778 (2004).

[31] P. Holmqvist, M. P. Lettinga, J. Buitenhuis, and J. K. G. Dhont, Langmuir 21, 10976 (2005).

[32] Y. D. Yan, J. K. G. Dhont, C. Smits, and H.N.W. Lekkerkerker, Physica A 202, 68 (1994).

[33] M. D. Haw, W. C. K. Poon, and P. N. Pusey, Phys. Rev. E 57, 6859 (1998).

[34] T. Palberg, W. Monch, J. Schwarz, and P. Leiderer, J. Chem. Phys. 102, 5082 (1995).

[35] T. Okubo, and H. Ishiki, J. Colloid Interface Sci. 211, 151 (2001).

[36] P. H. F. Hansen, T. Arnebrant, and L. Bergström, Colloid Polym. Sci. 279, 153 (2001).

[37] R. Blaak, S. Auer, D. Frenkel, and H. Löwen, J. Phys.: Condens. Matter 16, S3873 (2004); Phys. Rev. Lett. 93, 068303 (2004).

[38] S. Butler and P. Harrowell, Phys. Rev. E 52, 6424 (1995).

[39] A. V. Mokshin and J.-L. Barrat, Phys. Rev. E 77, 021505 (2008), and references therein.

[40] D. Reguera and J. M. Rubi, J. Chem. Phys. 119, 9888 (2003).

[41] S. Asakura and F. Oosawa, J. Chem. Phys. 22, 1255 (1954); A. Vrij, Pure Appl. Chem. 48, 471 (1976).

[42] R. de Rooij, A. A. Potatin, D. van den Ende, and J. Mellema, J. Chem. Phys. 99, 9213 (1993); 102, 5845 (1995).

[43] P. Snabre and P. Mills, J. Phys. III 6, 1811 (1996).

[44] R. Wessel and R. C. Ball, Phys. Rev. A 46, R3008 (1992).

[45] F. E. Torres, W. B. Russel, and W. R. Schowalter, J. Colloid Interface Sci. 142, 554 (1991).

[46] M. Doi and D. Chen, J. Chem. Phys. 90, 5271 (1989); 91, 2656 (1989).

[47] L. E. Silbert, J. R. Melrose, and R. C. Ball, Phys. Rev. E 56, 7067 (1997).

[48] L. E. Silbert, J. R. Melrose, and R. C. Ball, J. Rheol. 43, 673 (1999). 
[49] R. Buscall, J. Ian McGowan, and A. J. Morton-Jones, J. Rheol. 37, 621 (1993).

[50] J. J. Weis and D. Levesque, Adv. Polym. Sci. 185, 163 (2005).

[51] C. Selomulya, G. Bushell, R. Amal, and T. D. Waite, Langmuir 18, 1974 (2002).

[52] A. A. Potanin, R. De Rooij, D. Van den Ende, and J. Mellema, J. Chem. Phys. 102, 5845 (1995).

[53] V. Pryamitsyn and V. Ganesan, J. Chem. Phys. 122, 104906 (2005).

[54] J. R. Melrose and D. M. Heyes, J. Chem. Phys. 98, 5873 (1993).

[55] A. H. L. West, J. R. Melrose, and R. C. Ball, Phys. Rev. E 49, 4237 (1994).

[56] I. Dukovski and M. Muthukumar, J. Chem. Phys. 118, 6648 (2003).

[57] W. F. van Gunsteren and H. J. C. Berendsen, Mol. Phys. 45, 637 (1982).

[58] M. Ripoll, in Lecture Notes of the 37th IFF Spring School on Computational Condensed Matter Physics, edited by S. Blügel, G. Gompper, E. Koch, H. Müller-Krumbhaar, R. Spatscheck, and R. G. Winkler (Jülich, Germany, 2006).

[59] B. Dunweg, U. D. Schiller, and A. J. C. Ladd, Phys. Rev. E 76, 036704 (2007).

[60] Q. W. Lees and S. F. Edwards, J. Phys. C 5, 1921,(1972).

[61] V. Breedveld and A. J. Levine, Soft Mater. 1, 235 (2003).

[62] K. G. Soga, J. R. Melrose, and R. C. Ball, J. Chem. Phys. 108, 6026 (1998).

[63] K. G. Soga, J. R. Melrose, and R. C. Ball, J. Chem. Phys. 110, 2280 (1999).

[64] J. J. Cerdà, T. Sintes, C. M. Sorensen, and A. Chakrabarti,
Phys. Rev. E 70, 011405 (2004).

[65] D. Kashchiev and G. M. van Rosmalen, Cryst. Res. Technol. 38, 555 (2003).

[66] J. R. Melrose, Europhys. Lett. 19, 51 (1992).

[67] J. J. Cerdà, T. Sintes, C. M. Sorensen, and A. Chakrabarti, Phys. Rev. E 70, 051405 (2004).

[68] Note that this, in principle, does not imply that large seed crystals deposited on sheared systems for rates $\dot{\gamma}>\dot{\gamma}^{*}$, would unavoidably revert to single particles and could not trigger the crystal growth. The effects of shear in presence of crystal seeds are currently under study.

[69] S. J. Jung, R. Amal, and J. A. Raper, Powder Technol. 88, 51 (1996)

[70] Computations of $\left\langle s_{\text {break }}\right\rangle$ are quite involved but basically consist of keeping track of all clusters that have exceeded the size of $N=50$ particles at some instant. Clusters are labeled via a set of 25 , inner core particles. The labeling is updated at short time intervals $\Delta t \sim 0.1$ to maintain the labels as close as possible to the core of the cluster (preventing a loss of labeling via surface erosion). A cluster is considered to break when between two consecutive intervals $t$, and $t+\Delta t$, eleven or more labels are no longer present in the cluster.

[71] T. Hashimoto, K. Matsuzaka, E. Moses, and A. Onuki, Phys. Rev. Lett. 74, 126 (1995).

[72] E. K. Hobbie, S. Kim, and C. C. Han, Phys. Rev. E 54, R5909 (1996).

[73] Z. Shou and A. Chakrabarti, Phys. Rev. E 61, R2200 (2000).

[74] N. D. Vassileva, D. vd. Ende, F. Mugele, and J. Mellema, Langmuir 23, 2352 (2007). 